\title{
Fast Analysis of 2-D Electromagnetic Crystal Devices Using a Periodic Green Function Approach
}

\author{
Davy Pissoort, Eric Michielssen, Fellow, IEEE, Frank Olyslager, Fellow, IEEE, and Daniël De Zutter, Fellow, IEEE
}

\begin{abstract}
A novel integral equation-based method for simulating wave propagation in two-dimensional (2-D) electromagnetic crystal (EC) devices is presented. A small number of irregular defects aside, the targeted devices are obtained by removing cylinders from infinite, doubly periodic, and defectless electromagnetic crystals. Integral equations in terms of equivalent currents that reside on the surfaces of the voids left by the removed cylinders are constructed by using Green functions innate to the defectless electromagnetic crystal. The sparse system of equations that results upon discretizing these integral equations is solved efficiently by a multifrontal method. The scheme is ideally suited to extract electromagnetic crystal device $S$ parameters as it permits imposing modal excitations and exact absorbing boundary conditions. The scheme is applied to the analysis of two multiplexer-demultiplexer devices, a filter, and a bended EC waveguide, thereby demonstrating its versatility and computational efficiency.
\end{abstract}

Index Terms-Numerical analysis, periodic structures, photonic bandgap waveguides, photonic crystals.

\section{INTRODUCTION}

$\mathbf{R}$ ECENTLY, photonic or electromagnetic crystals (ECs) have been studied widely as their careful design enables the manipulation of optical/electromagnetic waves on spatial scales smaller than achievable by classical fiber structures [1]. Two-dimensional (2-D) ECs consist of parallel homogeneous dielectric cylinders residing on a periodic lattice in a homogeneous background. These ECs exhibit electromagnetic bandgaps, viz., ranges of frequencies for which no electromagnetic propagation is allowed. By removing/adding cylinders from/to an otherwise perfect EC, an EC device capable of supporting localized electromagnetic modes may result. This phenomenon can be exploited to create low-loss waveguides with sharp bends [2], [3], multiplexers [4]-[6], superprisms [7], etc.

In recent years, many computational schemes for simulating EC devices have been proposed. At present, the finitedifference time-domain method (FDTD) [8], which relates spatial samples of electromagnetic field variables on a staggered Cartesian grid via a temporal leapfrog scheme, is the

Manuscript received December 3, 2004; revised April 6, 2005.

D. Pissoort, F. Olyslager, and D. De Zutter are with the Electromagnetics Group, Department of Information Technology, Ghent University, Ghent, Belgium (e-mail: davy.pissoort@intec.ugent.be; frank.olyslager@intec.ugent.be; daniel.dezutter@intec.ugent.be).

E. Michielssen is with the Department of Electrical and Computer Engineering, University of Illinois at Urbana-Champaign, Urbana, IL 61801 USA (e-mail: emichiel@uiuc.edu).

Digital Object Identifier 10.1109/JLT.2005.850029 most popular among them. As the FDTD method operates directly in the time domain, it permits the wideband characterization of an EC device via a single simulation. Unfortunately, as ECs often contain small elements, their FDTD discretization and analysis require small spatial cells and time steps. Although the ensuing computational burden can be partially alleviated by using subcell models [9], FDTD methods remain computationally expensive, especially when high accuracies are required and phase dispersion is to be controlled. The eigenmode expansion method (EME) [10], [11] constitutes another frequently used technique for analyzing EC devices. The EME method slices up an EC device into sections with constant index profile along the propagation direction. Next, it expresses EC fields as a sum over each section's eigenmodes and determines the latter's expansion coefficients by mode matching at the section interfaces. While the EME method is very attractive when analyzing regular structures, its application becomes unwieldy when many different section types or curved structures are involved. The multiple scattering technique (MST) [12], [13] is a third popular method for analyzing EC devices. The MST solves integral equations in terms of equivalent currents that reside on the EC cylinders' surfaces. Often, the MST exploits the cylinders' circular nature by expanding surface currents in angular Fourier series, which permits their fields to be cast in terms of Bessel/Hankel functions. With this method, high accuracy can be obtained with only a few unknowns per cylinder. The MST's principal disadvantage is that it requires the solution of a dense linear system of equations whose dimension scales linearly with the number of cylinders. The cost of directly solving this system scales cubically in the numbers of cylinders. When using iterative solvers, this cost per iteration scales (nearly) linearly or quadratically in the number of cylinders depending on whether or not fast matrix-vector multiplication schemes are employed [14]. Unfortunately, the overall cost of the iterative solver also scales proportional to the total number of iterations required for its residual error to fall below a preset threshold. Numerical experiments have shown that for many realistic EC devices this number of iterations can be very high, especially when they contain waveguide components [15].

This paper describes a novel and fast MST for analyzing electromagnetic wave propagation in EC devices. A small number of defects aside, the targeted devices are obtained by removing cylinders from doubly periodic, defectless, and infinite ECs. The proposed method expressly cannot model radiation and mismatch effects caused by EC truncation, nor 


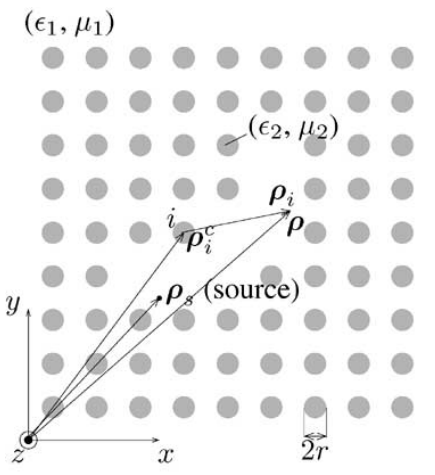

(a)

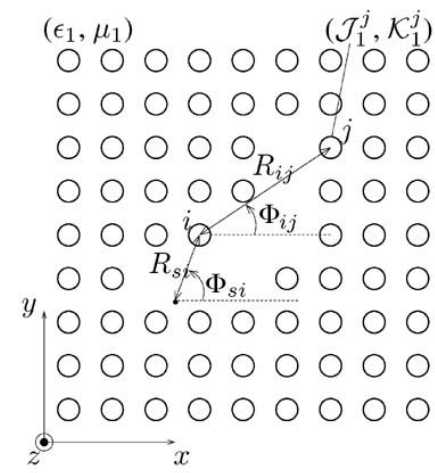

(b)

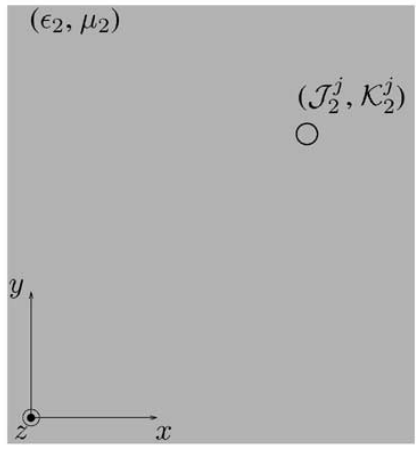

(c)

Fig. 1. Field-equivalence theorem applied to the simulation of finite EC devices using the free-space Green function approach. (a) Original configuration. (b) Exterior problem. (c) Interior problem.

can it analyze devices carved out of nonideal and perturbed ECs. Just like the conventional MST, the new MST solves integral equations in terms of equivalent currents that reside on cylindrical surfaces. However, contrary to the conventional MST, which associates currents with the surfaces of all physical cylinders that define the EC device and then subsequently describes their interactions using a free-space Green function, the new method considers unknown currents on the surfaces of fictitious removed cylinders and then models their interaction via a Green function innate to the surrounding infinite EC. For frequencies in the electromagnetic bandgap, this EC Green function decays exponentially with distance. Its precomputation can be achieved using the conventional MST by considering a centrally excited, finite, and small EC. Knowledge of the EC Green function permits the fast assembly of the novel MST's system of equations comprising a sparse interaction matrix and a localized excitation that can be solved rapidly by multifrontal methods. Precomputation of the EC Green function also permits calculation of the modes of the semiinfinite waveguides that terminate the EC device. Knowledge of these modes in turn can be used to compute the EC device's $S$ parameters as it enables the implementation of exact modal excitations and absorbing boundary conditions. Unfortunately, many EC devices do not fit the above mold. That is, they cannot be constructed by simply removing cylinders from an otherwise defectless and infinite EC as they contain defects, e.g., cylinders with center positions, radii, and/or material parameters that do not conform to those of the EC background. It will be shown that the proposed method applies to these structures as well, as they can be characterized by special Green functions that are low-rank perturbations of that of the defectless EC.

Notation: All sources and fields are assumed time harmonic with angular frequency $\omega$; temporal dependencies $e^{j \omega t}$ are suppressed.

\section{Analysis of EC Devices Using the Free-Space GREEN FUNCTION MST}

This section details the conventional MST for the characterization of finite EC devices comprising arbitrarily positioned dielectric/magnetic cylinders. This integral equation-based scheme uses a free-space Green function to describe interactions between equivalent currents on the cylinder surfaces. The field-equivalence principle [16] is invoked to elucidate symmetries between this conventional MST and the proposed novel MST (Section III).

Consider a finite 2-D EC device [Fig. 1(a)] comprising $N_{c}$ identical, infinite, and $z$-invariant, homogeneous, dielectric/ magnetic circular cylinders with radius $r$ and constitutive parameters (permittivity $\epsilon_{2}$, permeability $\mu_{2}$ ) that reside in a homogeneous background medium with constitutive parameters $\left(\epsilon_{1}, \mu_{1}\right)$. Let $\boldsymbol{\rho}=(\rho, \phi)$ denote a global position vector. Likewise, let $\boldsymbol{\rho}_{j}=\left(\rho_{j}, \phi_{j}\right)$ denote a local position vector with regard to the center $\boldsymbol{\rho}_{j}^{c}$ of cylinder $j, j=1, \ldots, N_{c}$. Let $E^{\mathrm{i}}(\boldsymbol{\rho}) \boldsymbol{u}_{z}$ denote the incident $\mathrm{TM}_{z}$-polarized electric field generated by impressed sources (assumed to reside outside the cylinders) in the absence of any cylinder. In the presence of the cylinders, the total field $E^{\mathrm{t}}(\boldsymbol{\rho}) \boldsymbol{u}_{z}$ is observed. The difference between the total and the incident fields is the scattered field $E^{\mathrm{s}}(\boldsymbol{\rho}) \boldsymbol{u}_{z}$, viz., $E^{\mathrm{t}}(\boldsymbol{\rho})=E^{\mathrm{i}}(\boldsymbol{\rho})+E^{\mathrm{s}}(\boldsymbol{\rho})$. To describe $E^{\mathrm{s}}(\boldsymbol{\rho})$ and $E^{\mathrm{t}}(\boldsymbol{\rho})$, two sets of equivalent electric and magnetic currents are introduced on the surface $S_{j}$ of every cylinder $j, j=1, \ldots, N_{c}$. These sets, labeled by subscripts $\alpha=1$ and 2 , relate to the total field on $S_{j}$ as

$$
\begin{aligned}
\mathcal{J}_{\alpha}^{j}(\boldsymbol{\rho}) \boldsymbol{u}_{z} & =-\left.s_{\alpha} \frac{j}{\omega \mu_{\alpha}} \frac{\partial E^{\mathrm{t}}(\boldsymbol{\rho})}{\partial \rho_{j}}\right|_{\boldsymbol{\rho} \in S_{j}} \delta\left(\rho_{j}-r\right) \boldsymbol{u}_{z} \\
\mathcal{K}_{\alpha}^{j}(\boldsymbol{\rho}) \boldsymbol{u}_{\phi_{j}} & =\left.s_{\alpha} E^{\mathrm{t}}(\boldsymbol{\rho})\right|_{\rho \in S_{j}} \delta\left(\rho_{j}-r\right) \boldsymbol{u}_{\phi_{j}}
\end{aligned}
$$

with $s_{1}=1$ and $s_{2}=-1$. Two assumptions follow from the field-equivalence theorem (Fig. 1):

i) The electric and magnetic currents $\sum_{j=1}^{N_{c}} \mathcal{J}_{1}^{j}(\rho) \boldsymbol{u}_{z}$ and $\sum_{j=1}^{N_{c}} \mathcal{K}_{1}^{j}(\boldsymbol{\rho}) \boldsymbol{u}_{\phi_{j}}$, when radiating alongside the impressed sources in an unbounded medium with constitutive parameters $\left(\epsilon_{1}, \mu_{1}\right)$, generate zero fields inside the (now fictitious) surface $S_{j}$ of every cylinder and the total electric field $E^{\mathrm{t}}(\boldsymbol{\rho}) \boldsymbol{u}_{z}$ outside all $S_{j}$. 
ii) The electric and magnetic currents $\mathcal{J}_{2}^{j}(\boldsymbol{\rho}) \boldsymbol{u}_{z}$ and $\mathcal{K}_{2}^{j}(\boldsymbol{\rho}) \boldsymbol{u}_{\phi_{j}}$, when radiating in an unbounded medium with constitutive parameters $\left(\epsilon_{2}, \mu_{2}\right)$, generate zero fields outside $S_{j}$ and $E^{\mathrm{t}}(\boldsymbol{\rho}) \boldsymbol{u}_{z}$ inside $S_{j}$.

Therefore, knowledge of $\mathcal{J}_{\alpha}^{j}(\boldsymbol{\rho})$ and $\mathcal{K}_{\alpha}^{j}(\boldsymbol{\rho})$ suffices to reconstruct all fields, scattered and/or total, both inside and outside the cylinders. If $E_{\alpha}^{\mathrm{s}, j} \boldsymbol{u}_{z}$ denotes the electric field radiated jointly by $\mathcal{J}_{\alpha}^{j}(\boldsymbol{\rho}) \boldsymbol{u}_{z}$ and $\mathcal{K}_{\alpha}^{j}(\boldsymbol{\rho}) \boldsymbol{u}_{\phi_{j}}$ in an unbounded medium with constitutive parameters $\left(\epsilon_{\alpha}, \mu_{\alpha}\right)$, then the above statements can be cast as

$$
\begin{array}{r}
E^{\mathrm{i}}(\boldsymbol{\rho})+\sum_{j=1}^{N_{c}} E_{1}^{\mathrm{s}, j}(\boldsymbol{\rho})=0 \text { for } \boldsymbol{\rho} \in S_{i}^{-}, \quad i=1, \ldots, N_{c} \\
E_{2}^{\mathrm{s}, j}(\boldsymbol{\rho})=0 \text { for } \boldsymbol{\rho} \in S_{j}^{+}, \quad j=1, \ldots, N_{c} .
\end{array}
$$

Here, $S_{j}^{-}$and $S_{j}^{+}$denote surfaces residing just inside and outside cylinder $j$, respectively. To solve (integral equations) (3) and $(4), \mathcal{J}_{\alpha}^{j}(\boldsymbol{\rho})$ and $\mathcal{K}_{\alpha}^{j}(\boldsymbol{\rho})$ are expanded into an angular Fourier series as

$$
\begin{gathered}
\mathcal{J}_{\alpha}^{j}(\boldsymbol{\rho})=s_{\alpha} \sum_{n=-K}^{K} \frac{C_{n} I_{n}^{j}}{2 \pi r} e^{j n \phi_{j}} \delta\left(\rho_{j}-r\right) \\
\mathcal{K}_{\alpha}^{j}(\boldsymbol{\rho})=s_{\alpha} \sum_{n=-K}^{K} \frac{C_{n} M_{n}^{j}}{2 \pi r} e^{j n \phi_{j}} \delta\left(\rho_{j}-r\right) .
\end{gathered}
$$

The constant

$$
C_{n}=\frac{k_{2} \mu_{1} J_{n}^{\prime}\left(k_{2} r\right)}{k_{2} \mu_{1} J_{n}\left(k_{1} r\right) J_{n}^{\prime}\left(k_{2} r\right)-k_{1} \mu_{2} J_{n}^{\prime}\left(k_{1} r\right) J_{n}\left(k_{2} r\right)}
$$

with $k_{\alpha}=\omega \sqrt{\epsilon_{\alpha} \mu_{\alpha}}$ is introduced to simplify the derivations and equations that follow. Because the cylinder radius $r$ is small compared with the wavelength and because the cylinders are assumed sufficiently separated from one another as well as from the impressed sources, the range of the modal index $n$ can always be restricted to $n=-K, \ldots,+K$ with $K$ as a small positive integer. It is readily verified that the scattered field $E_{\alpha}^{\mathrm{s}, j}(\rho)$ is given by (8) at the bottom of the page.

Here, $J_{n}(\cdot)$ is the $n$ th-order Bessel function of the first kind, and $H_{n}^{(2)}(\cdot)$ is the $n$ th-order Hankel function of the second kind.

Upon inserting expansion (8) into (3) and (4) and testing them using $T_{m}^{i}(\boldsymbol{\rho})=\left[1 / 2 \pi r J_{m}\left(k_{1} r\right)\right] e^{-j m \phi_{i}} \delta\left(\rho_{i}-r\right), i=$ $1, \ldots, N_{c} ; m=-K, \ldots, K$, the resulting set of linear equations can be solved for $I_{n}^{j}$ and $M_{n}^{j}, j=1, \ldots, N_{c} ; n=-K, \ldots, K$ by brute force. Alternatively, all the magnetic unknowns $M_{n}^{j}$ can be eliminated in favor of their electric counterparts $I_{n}^{j}$ by first solving (interior) (4), thereby leaving only (3) and electric unknowns $I_{n}^{j}$ to be considered. This strategy is adopted here. Inserting (8) with $\alpha=2$ into (4) yields

$$
\frac{I_{n}^{j}}{M_{n}^{j}}=-j \sqrt{\frac{\epsilon_{2}}{\mu_{2}}} \frac{J_{n}^{\prime}\left(k_{2} r\right)}{J_{n}\left(k_{2} r\right)}
$$

Using (9), (8) with $\alpha=1$ can be reexpressed as

$$
E_{1}^{\mathrm{s}, j}(\boldsymbol{\rho})=\sum_{n=-K}^{K} G_{n}\left(\boldsymbol{\rho}_{j}\right) I_{n}^{j}
$$

where $G_{n}\left(\boldsymbol{\rho}_{j}\right)$ given in (11), shown at the bottom of the page, is defined.

Quantity $G_{n}\left(\boldsymbol{\rho}_{j}\right)$ is the (generalized) free-space Green function for a Huygens source with distributed electric and magnetic components $\left(C_{n} / 2 \pi r\right) e^{j n \phi_{j}} \delta\left(\rho_{j}-r\right) \boldsymbol{u}_{z}$ and $\left(j C_{n} / 2 \pi r\right)$ $\sqrt{\left(\mu_{2} / \epsilon_{2}\right)}\left[J_{n}\left(k_{2} r\right) / J_{n}^{\prime}\left(k_{2} r\right)\right] e^{j n \phi_{j}} \delta\left(\rho_{j}-r\right) \boldsymbol{u}_{\phi_{j}}$ radiating jointly in an unbounded medium with constitutive parameters $\left(\epsilon_{1}, \mu_{1}\right)$.

To solve for the unknowns $I_{n}^{j}$, expansion (10) is inserted into (3), and the resulting equation is tested by $T_{m}^{i}(\rho), i=1, \ldots, N_{c}$; $m=-K, \ldots, K$, giving rise to the matrix equation

$$
\boldsymbol{Z I}=\boldsymbol{E}
$$

The entries of the matrix $\boldsymbol{Z}$ as well as the vector $\boldsymbol{E}$ are $Z_{m n}^{i j}=$ $\left\langle T_{m}^{i}(\rho), G_{n}\left(\rho_{j}\right)\right\rangle$ and $E_{m}^{i}=\left\langle T_{m}^{i}(\rho)-E^{\mathrm{i}}(\boldsymbol{\rho})\right\rangle$. Here $\langle\cdot, \cdot\rangle$ stands for the standard inner product. Making use of the addition theorem for the Hankel function [17, p. 232, eq. (5-103)], the

$$
E_{\alpha}^{\mathrm{s}, j}(\boldsymbol{\rho})= \begin{cases}-s_{\alpha} \sum_{n=-K}^{K}\left[\frac{\omega \mu_{\alpha}}{4} J_{n}\left(k_{\alpha} r\right) I_{n}^{j}+\frac{j k_{\alpha}}{4} J_{n}^{\prime}\left(k_{\alpha} r\right) M_{n}^{j}\right] C_{n} H_{n}^{(2)}\left(k_{\alpha} \rho_{j}\right) e^{j n \phi_{j}} & \text { if } \rho_{j}>r \\ -s_{\alpha} \sum_{n=-K}^{K}\left[\frac{\omega \mu_{\alpha}}{4} H_{n}^{(2)}\left(k_{\alpha} r\right) I_{n}^{j}+\frac{j k_{\alpha}}{4} H_{n}^{(2){ }^{\prime}}\left(k_{\alpha} r\right) M_{n}^{j}\right] C_{n} J_{n}\left(k_{\alpha} \rho_{j}\right) e^{j n \phi_{j}} & \text { if } \rho_{j}<r\end{cases}
$$

$$
G_{n}\left(\boldsymbol{\rho}_{j}\right)= \begin{cases}-\frac{\omega \mu_{1}}{4} H_{n}^{(2)}\left(k_{1} \rho_{j}\right) e^{j n \phi_{j}} & \text { if } \rho_{j}>r \\ -\frac{\omega \mu_{1}}{4} \frac{k_{2} \mu_{1} H_{n}^{(2)}\left(k_{1} r\right) J_{n}^{\prime}\left(k_{2} r\right)-k_{1} \mu_{2} H_{n}^{(2))^{\prime}}\left(k_{1} r\right) J_{n}\left(k_{2} r\right)}{k_{2} \mu_{1} J_{n}\left(k_{1} r\right) J_{n}^{\prime}\left(k_{2} r\right)-k_{1} \mu_{2} J_{n}^{\prime}\left(k_{1} r\right) J_{n}\left(k_{2} r\right)} J_{n}\left(k_{1} \rho_{j}\right) e^{j n \phi_{j}} & \text { if } \rho_{j}<r\end{cases}
$$




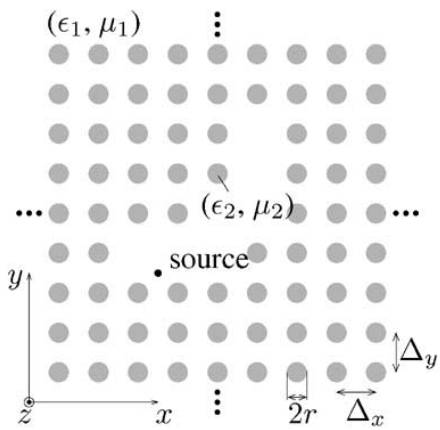

(a)

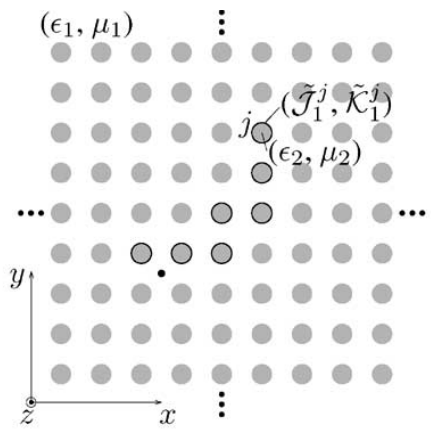

(b)

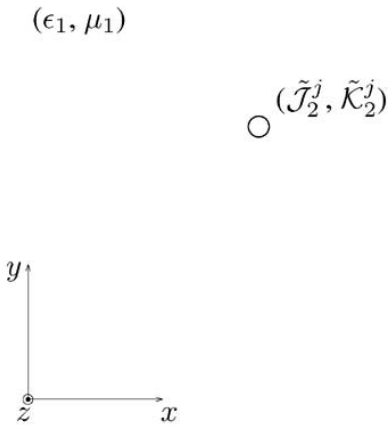

(c)

Fig. 2. Field-equivalence theorem applied to the simulation of an EC device using the EC Green function approach. (a) Original configuration. (b) Exterior problem. (c) Interior problem.

entries of the matrix $\boldsymbol{Z}$ are shown as in (13) at the bottom of the page.

Here $R_{i j}=\left|\boldsymbol{\rho}_{i j}^{c}\right|$ and $\Phi_{i j}=\arctan \left(\boldsymbol{\rho}_{i j}^{c} \cdot \boldsymbol{u}_{y} / \boldsymbol{\rho}_{i j}^{c} \cdot \boldsymbol{u}_{x}\right)$ are the length and angle with respect to the positive $x$-axis of the vector $\boldsymbol{\rho}_{i j}^{c}=\boldsymbol{\rho}_{j}^{c}-\boldsymbol{\rho}_{i}^{c}$ connecting the centers of the cylinders $i$ and $j$ [Fig. 1(b)]. For certain types of fields $E^{\mathrm{i}}(\rho)$, closed-form expressions of the entries of $\boldsymbol{E}$ also exist. For example, if $E^{\mathrm{i}}(\boldsymbol{\rho})$ is due to a unit strength electric line current located at $\boldsymbol{\rho}_{s}$, that is, if $E^{\mathrm{i}}(\boldsymbol{\rho})=-\left(\omega \mu_{1} / 4\right) H_{0}^{(2)}\left(k_{1}\left|\boldsymbol{\rho}-\boldsymbol{\rho}_{s}\right|\right)$, then use of the abovereferenced addition formula permits $E_{m}^{i}$ to be expressed as

$$
E_{m}^{i}=-\frac{\omega \mu_{1}}{4}(-1)^{m} H_{m}^{(2)}\left(k_{1} R_{s i}\right) e^{j m \Phi_{s i}} .
$$

Here, $R_{s i}=\left|\boldsymbol{\rho}_{s i}^{c}\right|$ and $\Phi_{s i}=\arctan \left(\boldsymbol{\rho}_{s i}^{c} \cdot \boldsymbol{u}_{y} / \boldsymbol{\rho}_{s i}^{c} \cdot \boldsymbol{u}_{x}\right)$ are the length and angle of the vector $\boldsymbol{\rho}_{s i}^{c}=\boldsymbol{\rho}_{i}^{c}-\boldsymbol{\rho}_{s}$ [Fig. 1(b)].

The above analysis can be easily repeated for $\mathrm{TE}_{z}$-polarized fields. The required modifications are principally twofold: 1) (3) and (4) are replaced by two similar equations stating that appropriate $z$-directed magnetic fields vanish inside or outside the surface $S_{j} ; 2$ ) magnetic and electric currents flow along $z$ and $\phi$, respectively.

The above "free-space Green function" MST does not preclude the characterization of EC devices with semi-infinite waveguide attachments. Indeed, such characterization can be achieved by terminating sufficiently long EC device waveguide appendages by absorbing/resistive sections, e.g., by perfectly matched layer-based absorbing boundary conditions for integral equation solvers [18]. The resulting scheme, potentially used in conjunction with computational de-embedding methods, then permits EC device $S$-parameter extraction.

Unfortunately, the above scheme's computational cost is high. The number of unknown electric current coefficients in the system (12) is $(2 K+1) N_{\mathrm{c}}$; here, $2 K+1$, the number of azimuthal harmonics per cylinder, does not depend on $N_{c}$, the extent of the crystal. As the latter grows, the cost of solving (12) using a direct solver thus scales as $O\left(N_{c}^{3}\right)$. By using classical iterative solvers, this cost can be reduced to $O\left(P N_{c}^{2}\right)$; here $P$ is the number of iterations. The cost can be further reduced to $O\left(P N_{c} \log N_{c}\right)$ or even $O\left(P N_{c}\right)$ by using fast Fourier transform (FFT) or multilevel fast multipole-based methods [14]. Unfortunately, numerical experiments have demonstrated that when the EC device contains waveguiding components, $P$ can be very high, even when a good preconditioner is used [15]. This precludes the application of this free-space Green function MST - even in conjunction with state of the art accelerators- to the analysis of all but the simplest EC devices.

\section{Analysis of EC Devices Using the EC GReEN FUNCTION MST}

This section details a novel and fast scheme for characterizing (semi-in)finite EC devices. A small number of defects aside, these devices are obtained by removing cylinders from an otherwise infinitely periodic and defectless EC. Contrary to the scheme described in the previous section, which characterized ECs by using a free-space Green function to describe interactions between equivalent currents on physical cylinders, the new scheme describes ECs by using a Green function innate to the defectless EC to model interactions between equivalent currents on removed cylinders.

\section{A. Formulation}

Consider a 2-D EC device [Fig. 2(a)] obtained by removing $N_{r}$ cylinders from an infinite and defectless EC comprising

$$
Z_{m n}^{i j}= \begin{cases}-\frac{\omega \mu_{1}}{4} \frac{k_{2} \mu_{1} H_{n}^{(2)}\left(k_{1} r\right) J_{n}^{\prime}\left(k_{2} r\right)-k_{1} \mu_{2} H_{n}^{(2))^{\prime}}\left(k_{1} r\right) J_{n}\left(k_{2} r\right)}{k_{2} \mu_{1} J_{n}\left(k_{1} r\right) J_{n}^{\prime}\left(k_{2} r\right)-k_{1} \mu_{2} J_{n}^{\prime}\left(k_{1} r\right) J_{n}\left(k_{2} r\right)} & \text { if } i=j \text { and } m=n \\ 0 & \text { if } i=j \text { and } m \neq n \\ -\frac{\omega \mu_{1}}{4} H_{n-m}^{(2)}\left(k_{1} R_{i j}\right) e^{j(m-n) \Phi_{i j}} & \text { if } i \neq j\end{cases}
$$


identical, infinite, and $z$-invariant, homogeneous, dielectric/ magnetic circular cylinders with radius $r$ and constitutive parameters $\left(\epsilon_{2}, \mu_{2}\right)$ that are spaced by $\Delta_{x}$ and $\Delta_{y}$ from center to center along the $x$ and $y$ directions and that reside in a homogeneous background medium with constitutive parameters $\left(\epsilon_{1}, \mu_{1}\right)$. The nomenclature in this section generally adheres to that used in Section II, with the exception that tildes appear on symbols referring to fields, currents, and surfaces of removed cylinders. It is assumed that $\omega$ lies within the EC bandgap. Let $\tilde{E}^{\mathrm{i}}(\boldsymbol{\rho}) \boldsymbol{u}_{z}$ denote the incident electric field generated by impressed sources that radiate in the presence of the defectless EC, viz., the infinite EC without any cylinders removed. Upon removal of the cylinders, the total field $\tilde{E}^{\mathrm{t}}(\boldsymbol{\rho}) \boldsymbol{u}_{z}$ is observed. The difference between the total and the incident fields is the scattered field $\tilde{E}^{\mathrm{s}}(\boldsymbol{\rho}) \boldsymbol{u}_{z}$, viz., $\tilde{E}^{\mathrm{t}}(\boldsymbol{\rho})=\tilde{E}^{\mathrm{i}}(\boldsymbol{\rho})+\tilde{E}^{\mathrm{s}}(\boldsymbol{\rho})$. To describe $\tilde{E}^{\mathrm{s}}(\boldsymbol{\rho})$ and $\tilde{E}^{\mathrm{t}}(\boldsymbol{\rho})$, two sets $(\alpha=1,2)$ of equivalent electric and magnetic currents are introduced on the surface $\tilde{S}_{j}$ of every removed cylinder $j, j=1, \ldots, N_{r}$. Both sets are related to the total field on $\tilde{S}_{j}$ by

$$
\begin{aligned}
\tilde{\mathcal{J}}_{\alpha}^{j}(\boldsymbol{\rho}) \boldsymbol{u}_{z} & =-\left.s_{\alpha} \frac{j}{\omega \mu_{1}} \frac{\partial \tilde{E}^{\mathrm{t}}(\boldsymbol{\rho})}{\partial \rho_{j}}\right|_{\boldsymbol{\rho} \in \tilde{S}_{j}} \delta\left(\rho_{j}-r\right) \boldsymbol{u}_{z} \\
\tilde{\mathcal{K}}_{\alpha}^{j}(\boldsymbol{\rho}) \boldsymbol{u}_{\phi_{j}} & =\left.s_{\alpha} \tilde{E}^{\mathrm{t}}(\boldsymbol{\rho})\right|_{\boldsymbol{\rho} \in \tilde{S}_{j}} \delta\left(\rho_{j}-r\right) \boldsymbol{u}_{\phi_{j}} .
\end{aligned}
$$

The following are found from the field-equivalence theorem (Fig. 2):

i) The electric and magnetic currents $\sum_{j=1}^{N_{r}} \tilde{\mathcal{J}}_{1}^{j}(\rho) \boldsymbol{u}_{z}$ and $\sum_{j=1}^{N_{r}} \tilde{\mathcal{K}}_{1}^{j}(\boldsymbol{\rho}) \boldsymbol{u}_{\phi_{j}}$, when radiating alongside the impressed sources in the unbounded and defectless EC, generate zero fields inside the surface $\tilde{S}_{j}$ of every cylinder that was originally removed and the total electric field $\tilde{E}^{\mathrm{t}}(\boldsymbol{\rho}) \boldsymbol{u}_{z}$ outside all $\tilde{S}_{j}$.

ii) The electric and magnetic currents $\tilde{\mathcal{J}}_{2}^{j}(\boldsymbol{\rho}) \boldsymbol{u}_{z}$ and $\tilde{\mathcal{K}}_{2}^{j}(\boldsymbol{\rho}) \boldsymbol{u}_{\phi_{j}}$, when radiating in an unbounded medium with constitutive parameters $\left(\epsilon_{1}, \mu_{1}\right)$, generate zero fields outside $\tilde{S}_{j}$ and $\tilde{E}^{\mathrm{t}}(\boldsymbol{\rho}) \boldsymbol{u}_{z}$ inside $\tilde{S}_{j}$.

If $\tilde{E}_{\alpha}^{\mathrm{s}, j}(\rho)$ denotes the amplitude of the $z$-directed electric field radiated jointly by $\tilde{\mathcal{J}}_{\alpha}^{j}(\boldsymbol{\rho}) \boldsymbol{u}_{z}$ and $\tilde{\mathcal{K}}_{\alpha}^{j}(\boldsymbol{\rho}) \boldsymbol{u}_{\phi_{j}}$ in the unbounded and defectless EC when $\alpha=1$ or in the unbounded medium with constitutive parameters $\left(\epsilon_{1}, \mu_{1}\right)$ when $\alpha=2$, then the above statements can be cast as

$$
\begin{array}{r}
\tilde{E}^{\mathrm{i}}(\boldsymbol{\rho})+\sum_{j=1}^{N_{r}} \tilde{E}_{1}^{\mathrm{s}, j}(\boldsymbol{\rho})=0 \text { if } \boldsymbol{\rho} \in \tilde{S}_{i}^{-}, \quad i=1, \ldots, N_{r} \\
\tilde{E}_{2}^{\mathrm{s}, j}(\boldsymbol{\rho})=0 \text { if } \boldsymbol{\rho} \in \tilde{S}_{j}^{+}, \quad j=1, \ldots, N_{r} .
\end{array}
$$

To solve (17) and (18), $\tilde{\mathcal{J}}_{\alpha}^{j}(\boldsymbol{\rho})$ and $\tilde{\mathcal{K}}_{\alpha}^{j}(\boldsymbol{\rho})$ are expanded as

$$
\begin{gathered}
\tilde{\mathcal{J}}_{\alpha}^{j}(\boldsymbol{\rho})=s_{\alpha} \sum_{n=-K}^{K} \frac{C_{n} \tilde{I}_{n}^{j}}{2 \pi r} e^{j n \phi_{j}} \delta\left(\rho_{j}-r\right) \\
\tilde{\mathcal{K}}_{\alpha}^{j}(\boldsymbol{\rho})=s_{\alpha} \sum_{n=-K}^{K} \frac{C_{n} \tilde{M}_{n}^{j}}{2 \pi r} e^{j n \phi_{j}} \delta\left(\rho_{j}-r\right) .
\end{gathered}
$$

Solving (18) now leads to

$$
\frac{\tilde{I}_{n}^{j}}{\tilde{M}_{n}^{j}}=-j \sqrt{\frac{\epsilon_{1}}{\mu_{1}}} \frac{J_{n}^{\prime}\left(k_{1} r\right)}{J_{n}\left(k_{1} r\right)} .
$$

Note the change in material index from 2 to 1 when comparing this ratio to that appearing in (9). This relationship permits $\tilde{E}_{1}^{\mathrm{s}, j}(\rho)$, the amplitude of the $z$-directed electric field radiated jointly by $\tilde{\mathcal{J}}_{1}^{j}(\rho)$ and $\tilde{\mathcal{K}}_{1}^{j}(\rho)$ in the defectless and unbounded EC, to be expressed solely in terms of electric unknowns as

$$
\tilde{E}_{1}^{\mathrm{s}, j}(\boldsymbol{\rho})=\sum_{n=-K}^{K} \tilde{G}_{n}\left(\boldsymbol{\rho}_{j}\right) \tilde{I}_{n}^{j} .
$$

Quantity $\tilde{G}_{n}\left(\boldsymbol{\rho}_{j}\right)$ is the (generalized) EC Green function for a Huygens source with distributed electric and magnetic components $\left(C_{n} / 2 \pi r\right) e^{j n \phi_{j}} \delta\left(\rho_{j}-r\right) \boldsymbol{u}_{z}$ and $\left(j C_{n} / 2 \pi r\right) \sqrt{\left(\epsilon_{1} / \mu_{1}\right)}$ $\left[J_{n}\left(k_{1} r\right) / J_{n}^{\prime}\left(k_{1} r\right)\right] e^{j n \phi_{j}} \delta\left(\rho_{j}-r\right) \boldsymbol{u}_{\phi_{j}}$ radiating jointly in the defectless and unbounded EC. Unfortunately, contrary to the developments in the previous section, no closed-form expressions for $\tilde{G}_{n}\left(\boldsymbol{\rho}_{j}\right)$ exist.

To solve for the unknowns $\tilde{I}_{n}^{j}$, expansion (22) is inserted into (17), and the resulting equation is tested by $\tilde{T}_{m}^{i}(\rho)=$ $\left[1 / 2 \pi r J_{m}\left(k_{2} r\right)\right] e^{-j m \phi_{i}} \delta\left(\rho_{i}-r\right)$, resulting in the matrix equation

$$
\tilde{\boldsymbol{Z}} \tilde{\boldsymbol{I}}=\tilde{\boldsymbol{E}}
$$

where $\tilde{Z}_{m n}^{i j}=\left\langle\tilde{T}_{m}^{i}(\boldsymbol{\rho}), \tilde{G}_{n}\left(\boldsymbol{\rho}_{j}\right)\right\rangle$ and $\quad \tilde{E}_{m}^{i}=\left\langle\tilde{T}_{m}^{i}(\boldsymbol{\rho}),-\tilde{E}^{\mathrm{i}}(\rho)\right\rangle$. Sections III-B and III-C detail techniques for evaluating the entries of $\tilde{\boldsymbol{Z}}$ and $\tilde{\boldsymbol{E}}$.

Matrix equations (12) and (23) differ in two important respects. First, for the vast majority of EC devices, $N_{r} \ll N_{\mathrm{c}}$. Second, whereas $\boldsymbol{Z}$ is dense, $\tilde{\boldsymbol{Z}}$ is essentially sparse. To see why, recall that $\tilde{G}_{n}\left(\boldsymbol{\rho}_{j}\right)$ is the EC Green function for a cylindrical Huygens source that radiates in the defectless and unbounded EC. Because $\omega$ is assumed to lie within the EC bandgap, $\tilde{G}_{n}\left(\boldsymbol{\rho}_{j}\right)$ decays exponentially with $\left|\boldsymbol{\rho}_{j}\right|$ 一this fact will also be demonstrated via numerical examples in Section IV. Therefore, each and every removed cylinder only interacts with its near neighbors, thereby rendering vanishingly small all entries of $\tilde{\boldsymbol{Z}}$ describing interactions between sufficiently separated removed cylinders. Consider, for example, the EC coupler depicted in Fig. 3. Fig. 4 shows the sparsity pattern of the corresponding interaction matrix $\tilde{\boldsymbol{Z}}$. Recently, significant advances in direct methods for inverting such sparse matrices have been reported, e.g., the multifrontal method by [19]. This method organizes the numerical factorization of a sparse matrix into a number of steps, each involving the formation of a dense smaller frontal matrix, followed by its partial factorization. The multifrontal method in the past has already been used for electromagnetic problems, e.g., in [20] and is adopted here to invert (23), thereby avoiding excessive iteration counts often encountered when analyzing EC devices using iterative solvers. 




Fig. 3. EC waveguide coupler.

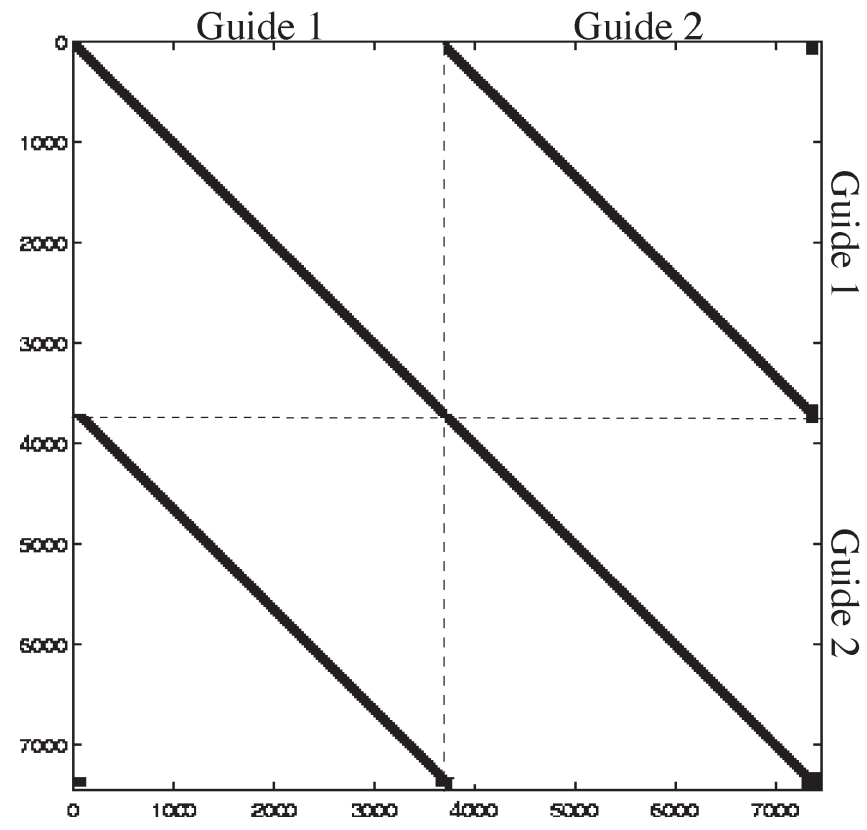

Fig. 4. Sparsity pattern of $\tilde{\boldsymbol{Z}}$ for the EC coupler of Fig. 3 with $l=1200 a$.

\section{B. Calculation of the EC Green Function $\tilde{G}_{n}(\rho)$ and the Entries of $\tilde{\mathbf{Z}}$}

The EC Green function $\tilde{G}_{n}\left(\boldsymbol{\rho}_{j}\right)$ for a cylindrical Huygens source that radiates in the defectless and unbounded EC cannot be evaluated analytically. Its numerical evaluation is aided by two facts, however. First, because the EC is periodic, $\tilde{G}_{n}\left(\boldsymbol{\rho}_{j}\right)$ does not depend on the cylinder index $j$. Second, as already mentioned above, $\tilde{G}_{n}(\rho)$ decays exponentially with $|\boldsymbol{\rho}|$. As a result, $\tilde{G}_{n}(\boldsymbol{\rho})$ can be evaluated using the conventional MST scheme detailed in Section II by considering a finite square EC of $N_{\mathrm{c}}=\left(2 N_{b}+1\right)^{2}$ cylinders wherein the central cylinder, which is assumed centered about the spatial origin $\boldsymbol{\rho}=\mathbf{0}$, is excited by a Huygens source with electric and magnetic components $\left(C_{n} / 2 \pi r\right) e^{j n \phi} \delta(\rho-r) \boldsymbol{u}_{z}$ and

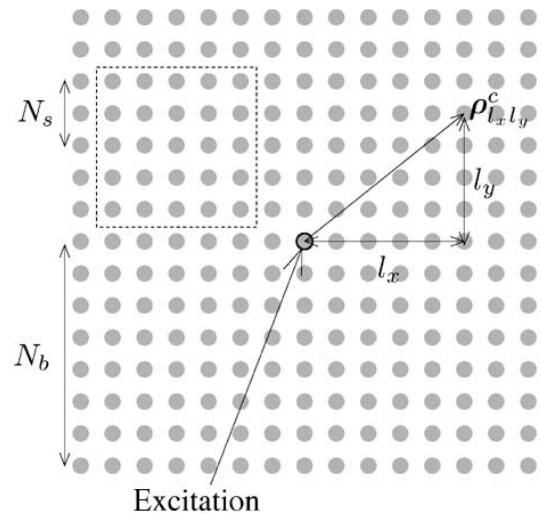

Fig. 5. Calculation of $\tilde{G}_{n}(\rho)$ using a centrally excited finite EC of $\left(2 N_{b}+1\right) \times\left(2 N_{b}+1\right)$ cylinders.

$\left(j C_{n} / 2 \pi r\right) \sqrt{\left(\epsilon_{1} / \mu_{1}\right)}\left[J_{n}\left(k_{1} r\right) / J_{n}^{\prime}\left(k_{1} r\right)\right] e^{j n \phi} \delta(\rho-r) \boldsymbol{u}_{\phi}$ (Fig. 5). It follows from (8), (18), and (21) that this source, when radiating in the background medium $\left(\epsilon_{1}, \mu_{1}\right)$, produces null fields for $\rho>r$ and

$$
E^{\mathrm{i}}(\rho)=-\frac{\omega \mu_{1}}{4} \frac{2 j C_{n}}{\pi k_{1} r J_{n}^{\prime}\left(k_{1} r\right)} J_{n}\left(k_{1} \rho\right) e^{j n \phi}
$$

for $\rho<r$. The parameter $N_{b}$ is chosen to be large enough such that outside this square EC the total field produced by this Huygens source essentially vanishes. Let $I_{m n}\left(l_{x}, l_{y}\right), l_{x}, l_{y}=$ $-N_{b}, \ldots, N_{b} ; m, n=-K, \ldots,+K$, denote the unknown describing the electric current's $m$ th harmonic that flows on the cylinder centered about $\boldsymbol{\rho}_{l_{x} l_{y}}^{c}=l_{x} \Delta_{x} \boldsymbol{u}_{x}+l_{y} \Delta_{y} \boldsymbol{u}_{y}$ in response to excitation by the above-described Huygens source (Fig. 5). Likewise, let $E_{m n}\left(l_{x}, l_{y}\right)$ denote the element of the excitation vector $\boldsymbol{E}$ in the formulation of Section II obtained by using the $m$ th harmonic testing function $T_{m}^{i}(\boldsymbol{\rho})$ on the fields produced by the Huygens source. It follows from (24) that

$$
E_{m n}\left(l_{x}, l_{y}\right)= \begin{cases}\frac{\omega \mu_{1}}{4} \frac{2 j C_{n}}{\pi k_{1} r J_{n}^{\prime}\left(k_{1} r\right)} & \text { if } l_{x}=l_{y}=0 \text { and } m=n \\ 0 & \text { otherwise. }\end{cases}
$$

Upon solving (12), with right-hand side (25) for $I_{m n}\left(l_{x}, l_{y}\right)$, it is seen that $\tilde{G}_{n}(\rho)$ can be expressed as (26), shown at the bottom of the page, with the position vector $\boldsymbol{\rho}_{l_{x} l_{y}}$ in the local cylindrical coordinate system centered about $\boldsymbol{\rho}_{l_{x} l_{y}}^{c}$. For future reference, let $\tilde{Z}_{m n}\left(l_{x}, l_{y}\right)$ denote the matrix element describing interactions

$$
\tilde{G}_{n}(\boldsymbol{\rho})= \begin{cases}\frac{\omega \mu_{2}}{4} \sum_{m=-K}^{K} \frac{2 j C_{m}}{\pi k_{2} r J_{m}^{\prime}\left(k_{2} r\right)} I_{m n}\left(l_{x}, l_{y}\right) J_{m}\left(k_{2} \rho_{l_{x} l_{y}}\right) e^{j m \phi_{l_{x} l_{y}}} & \text { if } \rho_{l_{x} l_{y}}<r \\ \sum_{l_{x}=-N_{b}}^{N_{b}} \sum_{l_{y}=-N_{b}}^{N_{b}} \sum_{m=-K}^{K} G_{m}\left(\boldsymbol{\rho}_{l_{x} l_{y}}\right) I_{m n}\left(l_{x}, l_{y}\right) & \text { if } \rho_{l_{x} l_{y}}>r \text { for all } l_{x}, l_{y}\end{cases}
$$


between modes $(m, n)$ on cylinders centered $l_{x} \Delta_{x}$ and $l_{y} \Delta_{y}$ apart along the $x$ and $y$ directions, respectively. It follows from (26) that $\tilde{Z}_{m n}\left(l_{x}, l_{y}\right)$ is

$$
\tilde{Z}_{m n}\left(l_{x}, l_{y}\right)=\frac{\omega \mu_{2}}{4} \frac{2 j C_{m}}{\pi k_{2} r J_{m}^{\prime}\left(k_{2} r\right)} I_{m n}\left(l_{x}, l_{y}\right)
$$

Note that all nonvanishing elements of the matrix $\tilde{\boldsymbol{Z}}$ in (23) are described by (27).

Matrix equation (12) for the centrally excited square EC with right hand side (25) can be solved efficiently by using an iterative, preconditioned, and FFT-accelerated method. It was shown in [15] that the use of a left "shielded-block preconditioner" effectively reduces the number of iterations to characterize finite and defectless ECs, this in contrary to EC devices with waveguiding structures. Instead of solving system $\boldsymbol{Z I}=\boldsymbol{E}$ to construct $\tilde{G}_{n}(\boldsymbol{\rho})$, system $\boldsymbol{M}_{s b} \boldsymbol{Z I}=\boldsymbol{M}_{s b} \boldsymbol{E}$ is solved. The preconditioner $\boldsymbol{M}_{s b}$ is formed by selecting, out of $\boldsymbol{Z}$, for each and every cylinder, a small $2 N_{s}+1$ by $2 N_{s}+1$ interaction matrix $\left(N_{s}<N_{b}\right)$ of nearest interactions (Fig. 5). This reduced interaction matrix is inverted and the rows corresponding to the targeted cylinder extracted and inserted into $\boldsymbol{M}_{s b}$.

\section{Computation of the EC-Impressed Field $\tilde{E}^{\mathrm{i}}(\boldsymbol{\rho})$ and the Entries of $\tilde{\boldsymbol{E}}$-Modal Excitation/Absorbing Boundary Conditions of the Semi-Infinite EC}

The $z$-component of the electric field generated by impressed sources that radiate in the presence of the defectless EC $\tilde{E}^{\mathrm{i}}(\rho)$ cannot be evaluated analytically. If the impressed source is a $z$-directed electric line current at $\boldsymbol{\rho}_{s}$ (assumed to reside outside all the physical and removed cylinders), then $\tilde{E}^{\mathrm{i}}(\boldsymbol{\rho})$ can, just like $\tilde{G}_{n}(\boldsymbol{\rho})$, be computed using the free-space Green function MST of Section II. Indeed, $\widetilde{E}^{\mathrm{i}}(\rho)$ comprises $E^{\mathrm{i}}(\boldsymbol{\rho})=-\left(\omega \mu_{1} / 4\right) H_{0}^{(2)}\left(k_{1}\left|\boldsymbol{\rho}-\boldsymbol{\rho}_{s}\right|\right)$ plus the field scattered by the cylinders. The latter can be calculated using (10), following the computation of the currents on cylinders near the source. To this end, consider a finite EC comprising $N_{c}=\left(2 N_{b}+1\right)^{2}$ cylinders centered about the origin, which is also assumed near $\boldsymbol{\rho}_{s}$. Let $I_{m s}\left(l_{x}, l_{y}\right)$ denote the electric current's $m$ th harmonic that flows on the cylinder centered about $\boldsymbol{\rho}_{l_{r} l_{y}}^{c}$ in this finite EC when excited with the field of the electric line current. These coefficients $I_{m s}\left(l_{x}, l_{y}\right)$ are obtained by solving (12) with the entries of the excitation vector $\boldsymbol{E}$ given by (14), which leads to (28), shown at the bottom of the page.

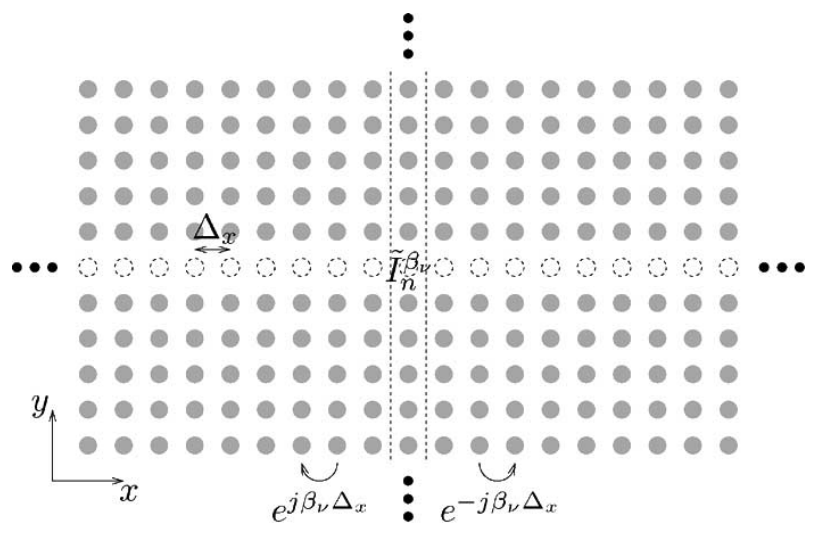

Fig. 6. Calculation of an EC waveguide's eigenmodes.

Hence, the entries of the excitation vector $\tilde{\boldsymbol{E}}$ are

$$
\tilde{E}_{m s}\left(l_{x}, l_{y}\right)=\frac{\omega \mu_{2}}{4} \frac{2 j C_{m}}{\pi k_{2} r J_{m}^{\prime}\left(k_{2} r\right)} I_{m s}\left(l_{x}, l_{y}\right) .
$$

The above EC Green function MST with line source excitation can be used to characterize the effects of semi-infinite waveguides attached to finite EC devices by terminating sufficiently long waveguide appendages by resistive sections. The scheme, when used in conjunction with computational deembedding techniques, then permits EC $S$-parameter extraction.

The EC Green function scheme however allows for an intriguing alternative to find the EC's $S$ parameters, as it allows for an easy identification of the propagating EC defect/ waveguide modes and their subsequent use as modal excitations and boundary conditions when constructing system (23). To see how the EC Green function method can be used to find an EC waveguide's eigenmodes, let $\tilde{E}_{\nu}(\boldsymbol{\rho}) \boldsymbol{u}_{z}$ denote the $z$-directed electric field of the $\nu$ th forward propagating eigenmode in an infinitely long, $x$-directed EC waveguide. To simplify the ensuing discussion, assume that this waveguide is formed by removing a single $x$-directed row of cylinders from an unbounded, defectless EC (Fig. 6). According to the Floquet-Bloch theorem, $\tilde{E}_{\nu}(\rho)$ satisfies

$$
\tilde{E}_{\nu}(\boldsymbol{\rho})=\tilde{e}_{\nu}(\boldsymbol{\rho}) e^{-j \beta_{\nu} x}
$$

where $\tilde{e}_{\nu}\left(\boldsymbol{\rho}+\Delta_{x} \boldsymbol{u}_{x}\right)=\tilde{e}_{\nu}(\boldsymbol{\rho})$ and $\beta_{\nu}$ is the mode's (assumed real) propagation constant. Of course, $\tilde{E}_{\nu}(\rho)$ can be characterized in terms of equivalent electric and magnetic currents on the surfaces of the removed cylinders. Moreover, the evolution of these currents' amplitudes along the waveguide channel is

$$
\tilde{E}^{\mathrm{i}}(\rho)= \begin{cases}\frac{\omega \mu_{2}}{4} \sum_{m=-K}^{K} \frac{2 j C_{m}}{\pi k_{2} r J_{m}^{\prime}\left(k_{2} r\right)} I_{m s}\left(l_{x}, l_{y}\right) J_{m}\left(k_{2} \rho_{l_{x} l_{y}}\right) e^{j m \phi_{l_{x} y}} & \text { if } \rho_{l_{x} l_{y}}<r \\ E^{\mathrm{i}}(\boldsymbol{\rho})+\sum_{l_{x}=-N_{b}}^{N_{b}} \sum_{l_{y}=-N_{b}}^{N_{b}} \sum_{m=-K}^{K} G_{m}\left(\boldsymbol{\rho}_{l_{x} l_{y}}\right) I_{m s}\left(l_{x}, l_{y}\right) & \text { if } \rho_{l_{x} l_{y}}>r \text { for all } l_{x}, l_{y}\end{cases}
$$




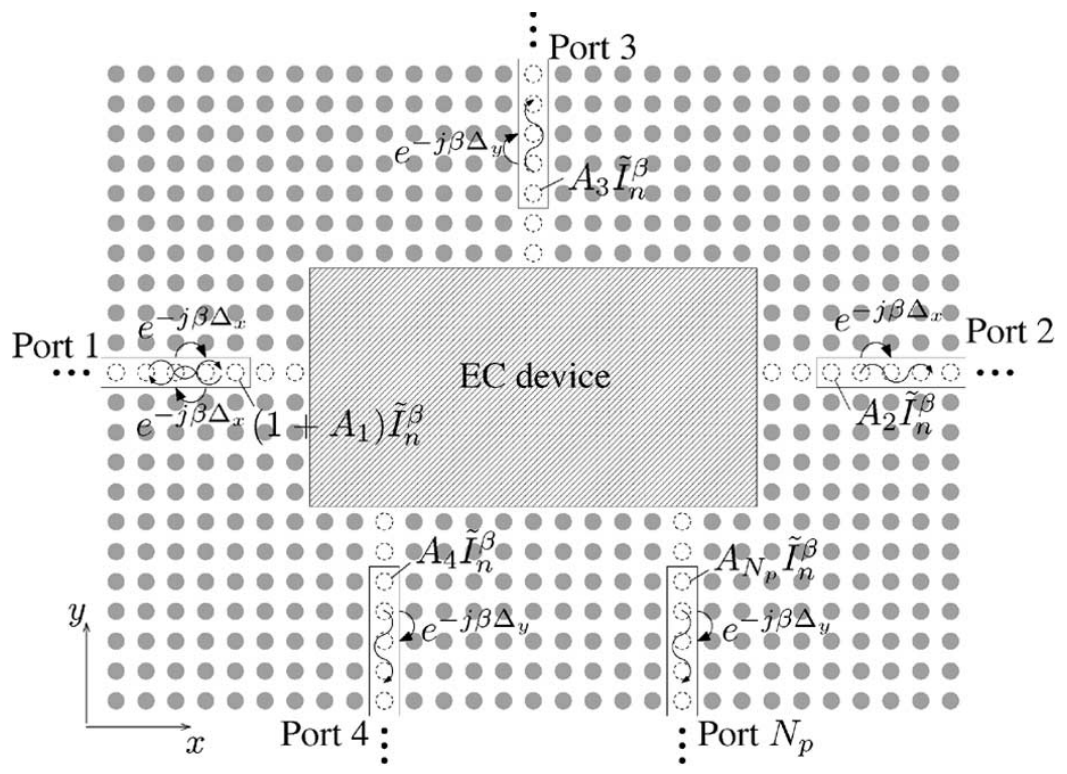

Fig. 7. Modal absorbing boundary conditions and excitation.

dictated by that of the fields they were derived from, viz., (30), and hence equivalent currents in only one unit cell suffice to fully characterize the modal field distribution. Mirroring the derivations in Section III-A, it follows that the electric current expansion coefficients $\tilde{I}_{n}^{\beta_{\nu}}, n=-K, \ldots, K$ on the removed cylinder in the unit cell satisfy

$\sum_{n=-K}^{K}\left[\sum_{l_{x}=-N_{b}}^{N_{b}} \tilde{Z}_{m n}\left(l_{x}, 0\right) e^{-j \beta_{\nu} l_{x} \Delta_{x}}\right] \tilde{I}_{n}^{\beta_{\nu}}=0$,

$$
\text { for } m=-K, \ldots, K \text {. }
$$

Note that in (31) only currents of $2 N_{b}+1$ cells symmetrically placed to the left and right of the cell in which currents $\tilde{I}_{n}^{\beta_{\nu}}$ are measured are accounted for-this once again is possible only because $\omega$ lies in the EC bandgap. Setting the $\beta_{\nu}$-dependent determinant of the matrix derived from system (31) to zero allows the waveguide's propagation constants to be determined. Once the propagation constants of the various modes are found, their transverse profiles are described by the null space of (31) with fixed $\beta_{\nu}$.

Once the waveguide modes have been characterized, they can be used, within the framework of the EC Green function MST, to terminate/excite the EC device in/from semi-infinite waveguides. To illustrate this procedure, consider an EC device connected to a set of $N_{p}$ identical semi-infinite waveguides (Fig. 7) - to simplify the presentation, the assumption that these waveguides are formed by removing one row of cylinders from the EC remains in effect. To implement an exact absorbing boundary condition, assume that these waveguides support only one propagating mode with propagation constant $\beta$. Far enough from all discontinuities, the fields propagating away from the EC device in each of these waveguides are adequately described by a single outward propagating mode. Let the currents on the first cylinder in the waveguide where this behavior is believed to hold true be denoted by $A_{j} \tilde{I}_{n}^{\beta} ; A_{j}$ is referred to as the waveguide $j$ outgoing mode's amplitude. This cylinder, along with all that follow, is referred to as the "cylinders of port $j$ "- in what follows, they are to be distinguished from the $N_{r}$ "regularly removed cylinders" that define the EC device. The current expansion coefficients for the cylinders of port $j$ are related to one another by the Floquet-Bloch theorem (30), as indicated in Fig. 7. The unknowns now comprise $(2 K+1) N_{r}$ electric current unknowns $\tilde{I}_{n}^{j}, j=1, \ldots, N_{r} ; j=-K, \ldots, K$, and $N_{p}$ amplitudes $A_{j}, j=1, \ldots, N_{p}$, of the outgoing modes. To account for the outgoing modal fields, (17) is now changed to

$$
\begin{aligned}
\tilde{E}^{\mathrm{i}}(\boldsymbol{\rho})+\sum_{j=1}^{N_{r}} \tilde{E}_{1}^{\mathrm{s}, j}(\boldsymbol{\rho})+\sum_{j=1}^{N_{p}} \tilde{E}_{1}^{\mathrm{s}, p_{j}}(\boldsymbol{\rho})=0, \\
\quad \text { for } \boldsymbol{\rho} \in \tilde{S}_{i}^{-}, \quad i=1, \ldots, N_{r}+N_{p} .
\end{aligned}
$$

Here, $\tilde{E}_{1}^{s, p_{j}}(\rho)$ represents the sum of the fields produced by all the currents on the cylinders of port $j$. A first set of $(2 K+1) N_{r}$ equations are obtained by testing (32) on the surfaces of all $N_{r}$ regularly removed cylinders with $\tilde{T}_{m}^{i}(\boldsymbol{\rho}), i=$ $1, \ldots, N_{r} ; m=-K, \ldots, K$. The matrix elements describing interactions between regularly removed cylinders resulting from this procedure are still described by (27). Assuming that port $j$ consists of a $+x$-directed waveguide, weighting $\tilde{E}_{1}^{\mathrm{s}, p_{j}}(\boldsymbol{\rho})$ on a cylinder whose center is located $-l_{x} \Delta_{x}\left(l_{x}>0\right)$ and $l_{y} \Delta_{y}$ away from the center of the first cylinder of port $j$ along the $x$ and the $y$ direction, respectively, yields a contribution

$$
\begin{array}{r}
\tilde{Z}_{m p_{j}}\left(-l_{x}, l_{y}\right) A_{j}=A_{j} \sum_{l_{s}=0}^{N_{b}} \sum_{n=-K}^{K} \tilde{Z}_{m n}\left(-l_{x}+l_{s}, l_{y}\right) \tilde{I}_{n}^{\beta} e^{-j \beta l_{s} \Delta_{x}} \\
m=-K, \ldots, K
\end{array}
$$


where $\tilde{Z}_{m p_{i}}\left(l_{x}, l_{y}\right)$ denotes the effective matrix element describing the interaction of port $p_{j}$ with a regularly removed cylinder. A second set of $N_{p}$ equations is found by weighting (32) on the surface of the first cylinder of every port with $\widetilde{T}_{0}^{i}(\rho)$. Weighting the second term of (32) leads to the matrix elements $\tilde{Z}_{0 n}\left(l_{x}, l_{y}\right)$, while weighting the third term of (32) again leads to the effective matrix elements $\tilde{Z}_{0 p_{j}}\left(l_{x}, l_{y}\right)$ defined in (33). Similar arguments and expressions can be proffered for ports associated with waveguides leaving the EC device in other directions.

To illustrate how to impose modal excitations within the EC Green function MST framework, assume that the EC device is excited by a propagating mode coming from the semiinfinite waveguide 1 . The incident field $\tilde{E}^{\mathrm{i}}(\boldsymbol{\rho})$ is the sum of the fields produced by all the currents on the cylinders of port 1 , with the current expansion coefficients on the first cylinder equal to $\tilde{I}_{n}^{\beta}$ and those for consecutive cylinders related by the Floquet-Bloch theorem. For example, assume that port 1 is $-x$ directed, which means that a mode that is incoming into the EC device propagates in the $+x$-direction. Following the same reasoning as for the absorbing boundary condition, it can be readily seen that weighting the incident field on the surface of a cylinder whose center is $l_{x} \Delta_{x}$ and $l_{y} \Delta_{y}$ apart from that of the first cylinder of port 1 in the $x$ - and the $y$-direction gives the following entries for the excitation vector $\tilde{\boldsymbol{E}}$

$$
\begin{array}{r}
\tilde{E}_{m}\left(l_{x}, l_{y}\right)=\sum_{l_{s}=0}^{N_{b}} \sum_{n=-K}^{K} \tilde{Z}_{m n}\left(l_{x}-l_{s}, l_{y}\right) \tilde{I}_{n}^{\beta} e^{-j \beta l_{s} \Delta_{x}} \\
m=-K, \ldots, K .
\end{array}
$$

Note that when all the semi-infinite waveguides are identical, the amplitudes of the outgoing modes correspond immediately with the $S$ parameters of the EC device: $S_{1 j}=A_{j}$. The reasoning followed in this section can be generalized to waveguides with more than one cylinder across and/or to different types of semiinfinite waveguide appendages.

\section{Special Defects in the EC}

The scheme detailed in Sections III-A, III-B, and III-C only permits the characterization of EC devices that are obtained by removing cylinders from defectless ECs. However, many practical EC devices also contain defects other than removed cylinders. For example, filters sometimes contain special cylinders whose radius and/or constitutive parameters differ from those of the background EC elements to create resonant cavities. And EC devices with bended waveguides often contain special cylinders that are displaced from the background EC lattice to minimize reflections. In this section, it will be shown that ECs with (a few) special defects can be treated by a simple extension of the scheme of Sections III-A, III-B, and III-C.

Generally speaking, the scheme of Section III-A applies to ECs with special defects, provided that their presence is reflected in the Green function. That is, when sources and/or observers reside near a special defect, a Green function different from that computed in Section III-B must be used (Fig. 8).

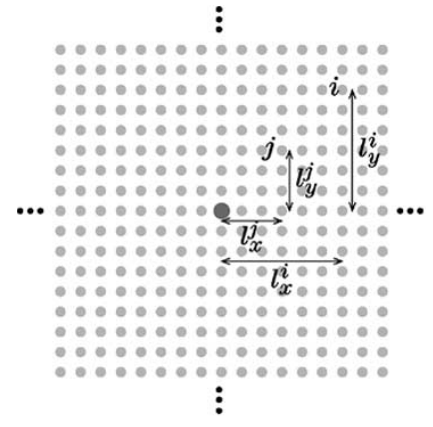

(a) (b)

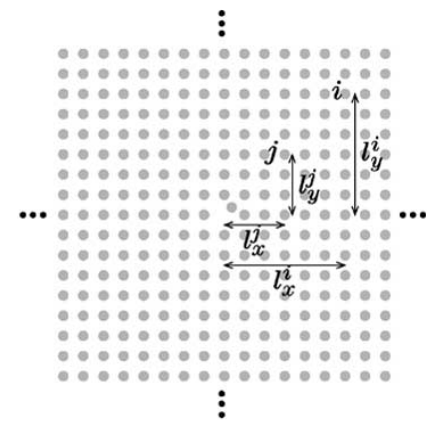

Fig. 8. Special Green functions.

Recall that the scheme described in Section III-B for computing the EC Green function involved the construction (and conceptual inversion) of a conventional MST interaction matrix $\boldsymbol{Z}$ for a finite, square, and defectless EC. Of course, the computation of the Green function for an EC with special defects can be effected by very similar techniques, provided that the finite EC considered now contains the special defect. Fortunately, the conventional MST interaction matrix for a finite EC with special defect further denoted as $\hat{\boldsymbol{Z}}$ is a low-rank update of the interaction matrix for the defectless finite $\mathrm{EC}$

$$
\hat{\mathbf{Z}}=\boldsymbol{Z}+\boldsymbol{U} \boldsymbol{V}^{T}
$$

According to the Sherman-Morrison formula [21], $\hat{\boldsymbol{Y}}=\hat{\boldsymbol{Z}}^{-\mathbf{1}}$ is also a low-rank update of $\boldsymbol{Y}=\boldsymbol{Z}^{-\mathbf{1}}$

$$
\hat{\boldsymbol{Y}}=\boldsymbol{Y}-\boldsymbol{Y} \boldsymbol{U}\left(\mathbf{1}+\boldsymbol{V}^{T} \boldsymbol{Y} \boldsymbol{U}\right)^{-1} \boldsymbol{V}^{T} \boldsymbol{Y} .
$$

In (36), $\mathbf{1}$ stands for the identity matrix. Therefore, $\hat{\boldsymbol{Y}}$ can be calculated very fast without resorting to solving a new linear system. Below, the vectors $\boldsymbol{U}$ and $\boldsymbol{V}$ are described for two important cases: special defects involving 1) a cylinder with modified constitutive parameters and/or radius and 2) a cylinder displaced from its background lattice position. To simplify the notation, only cases involving one special defect and ECs in which currents on cylinders are modeled by one unknown $(K=0)$ are considered; extensions to more complicated scenarios are straightforward.

1) A Cylinder With Modified Constitutive Parameters and/or Radius Situated on a Regular Lattice Node [Fig. 8(a)]: In this case, it follows from (13) that the matrices $\hat{\mathbf{Z}}$ and $\boldsymbol{Z}$ only differ by one element, namely the self-interaction of the special cylinder. Suppose the index of this cylinder is 1 and that this cylinder corresponds with the central cylinder in the finite EC that is used to calculate the Green function. With $\Delta=\hat{Z}_{00}^{11}-Z_{00}^{11}, \boldsymbol{U}$ and $\boldsymbol{V}$ read

$$
\boldsymbol{U}=\left(\begin{array}{c}
1 \\
0 \\
\vdots \\
0
\end{array}\right), \quad \boldsymbol{V}=\left(\begin{array}{c}
\Delta \\
0 \\
\vdots \\
0
\end{array}\right)
$$


Because the Green function is calculated using an iterative method, $\boldsymbol{Y}$ is not explicitly known. In Section III-B, it was shown that the excitation vector $\boldsymbol{E}$ completely consists of zeros except for the element corresponding with the central cylinder (cylinder 1). This means that $\boldsymbol{I}$ is proportional to the first column of $\boldsymbol{Y}$. If $i$ is the index of the cylinder centered about $l_{x}^{i} \Delta_{x} \boldsymbol{u}_{x}+l_{y}^{i} \Delta_{y} \boldsymbol{u}_{y}$, then

with

$$
Y_{00}^{i 1}=D_{0} I_{00}\left(l_{x}^{i}, l_{y}^{i}\right)
$$

$$
D_{0}=\frac{4}{\omega \mu_{1}} \frac{\pi k_{1} r J_{0}^{\prime}\left(k_{1} r\right)}{2 j C_{0}}
$$

Although the iterative solution of (13) only gives the first column of $\boldsymbol{Y}$, all the elements of this matrix are known if it is kept in mind that in fact the Green function for an infinite EC is calculated. Indeed, $Y_{00}^{i j}$ is proportional to the current on cylinder $i$, when the excitation is on cylinder $j$. For an infinite EC, the solution corresponding to the excitation on cylinder $j$ can be derived from that of exciting cylinder 1 by a shift operation, and hence

$$
Y_{00}^{i j}=D_{0} I_{00}\left(l_{x}^{i}-l_{x}^{j}, l_{y}^{i}-l_{y}^{j}\right) .
$$

Suppose that $\tilde{Z}_{00}^{i j}$, the element in the sparse interaction matrix of the new method that describes the interaction between cylinder $i$ and cylinder $j$ that reside $\left(l_{x}^{i} \Delta_{x}, l_{y}^{i} \Delta_{y}\right)$ and $\left(l_{x}^{j} \Delta_{x}, l_{y}^{j} \Delta_{y}\right)$ apart along the $x$ - and $y$-direction from the special cylinder, has to be calculated (Fig. 8). Note that $\boldsymbol{Y} \boldsymbol{U}$ corresponds with the first column of $\boldsymbol{Y}$ and that $\boldsymbol{V}^{T} \boldsymbol{Y}$ corresponds with $\Delta$ times the first row of $\boldsymbol{Y}$. According to (36)

$$
\hat{Y}_{00}^{i j}=Y_{00}^{i j}-Y_{00}^{i 1}\left(1+\Delta Y_{00}^{11}\right)^{-1} \Delta Y_{00}^{1 j} .
$$

The combination of (27), (40), and (41) yields

$$
\begin{array}{r}
\tilde{Z}_{00}^{i j}=\frac{\omega \mu_{2}}{4} \frac{2 j C_{0}}{\pi k_{2} r J_{0}^{\prime}\left(k_{2} r\right)}\left[I_{00}\left(l_{x}^{i}-l_{x}^{j}, l_{y}^{i}-l_{y}^{j}\right)+I_{00}\left(l_{x}^{i}, l_{y}^{i}\right)\right. \\
\left.\times \frac{\Delta D_{0}}{1+\Delta D_{0} I_{00}(0,0)} I_{00}\left(-l_{x}^{j},-l_{y}^{j}\right)\right] .
\end{array}
$$

2) A Cylinder Displaced From Its Background Lattice Position (With the Same Constitutive Parameters and Radius as Those of the Background EC Lattice) [Fig. 8(b)]: Now, $\hat{\mathbf{Z}}$ and $\boldsymbol{Z}$ differ by the whole first column and row, except for the diagonal element. So, $\boldsymbol{U}$ and $\boldsymbol{V}$ can be expressed as

$$
\begin{aligned}
& \boldsymbol{U}=\left(\begin{array}{cc}
1 & 0 \\
0 & \Delta_{21} \\
\vdots & \vdots \\
0 & \Delta_{N_{\mathrm{c}}}
\end{array}\right)=\left(\begin{array}{ll}
\boldsymbol{U}_{1} & \boldsymbol{U}_{2}
\end{array}\right) \\
& \boldsymbol{V}=\left(\begin{array}{cc}
0 & 1 \\
\Delta_{12} & 0 \\
\vdots & \vdots \\
\Delta_{1 N_{\mathrm{c}}} & 0
\end{array}\right)=\left(\begin{array}{ll}
\boldsymbol{V}_{1} & \boldsymbol{V}_{2}
\end{array}\right)
\end{aligned}
$$

with $\Delta_{i j}=\hat{Z}_{00}^{i j}-Z_{00}^{i j}$. The calculation of $\boldsymbol{Y} \boldsymbol{U}_{1}$ and $\boldsymbol{V}_{2}^{T} \boldsymbol{Y}$ has already been treated above. Let $U_{2 i}$ denote the $i$ th element of the vector $\boldsymbol{U}_{2}$. To calculate $\boldsymbol{Y} \boldsymbol{U}_{2}$, note that

$$
\boldsymbol{Y}\left(\begin{array}{c}
U_{21} \\
U_{22} \\
\vdots \\
\vdots \\
U_{2 N_{\mathrm{c}}}
\end{array}\right)=U_{21} \boldsymbol{Y}\left(\begin{array}{c}
1 \\
0 \\
\vdots \\
\vdots \\
0
\end{array}\right)+U_{22} \boldsymbol{Y}\left(\begin{array}{c}
0 \\
1 \\
0 \\
\vdots \\
0
\end{array}\right)+\ldots+U_{2 N_{\mathrm{c}}} \boldsymbol{Y}\left(\begin{array}{c}
0 \\
\vdots \\
\vdots \\
0 \\
1
\end{array}\right)
$$

With $U_{2}\left(l_{x}^{i}, l_{y}^{i}\right)=U_{2 i}$ and following the above reasoning, it is seen that the $i$ th element of $\boldsymbol{Y} \boldsymbol{U}_{2}$ is nothing but

$$
\left(\boldsymbol{Y} \boldsymbol{U}_{2}\right)_{i}=D_{0} \sum_{j=1}^{N_{\mathrm{c}}} U_{2}\left(l_{x}^{j}, l_{y}^{j}\right) I_{00}\left(l_{x}^{i}-l_{x}^{j}, l_{y}^{i}-l_{y}^{j}\right) .
$$

Similarly

$$
\left(\boldsymbol{V}_{1}^{T} \boldsymbol{Y}\right)_{i}=D_{0} \sum_{j=1}^{N_{\mathrm{c}}} V_{1}\left(l_{x}^{j}, l_{y}^{j}\right) I_{00}\left(l_{x}^{j}-l_{x}^{i}, l_{y}^{j}-l_{y}^{i}\right)
$$

with $V_{1}\left(l_{x}^{i}, l_{y}^{i}\right)=V_{1 i}$. Equations (45) and (46) show that $\boldsymbol{Y} \boldsymbol{U}_{2}$ and $\boldsymbol{V}_{1}^{T} \boldsymbol{Y}$ are discrete 2-D convolutions and, hence, can be calculated quickly using a 2-D FFT. However, if one wants to know $\boldsymbol{Y} \boldsymbol{U}_{2}$ and $\boldsymbol{V}_{1}^{T} \boldsymbol{Y}$ inside a square EC of $\left(2 N_{b}+1\right) \times$ $\left(2 N_{b}+1\right)$ cylinders, $\boldsymbol{U}_{2}$ and $\boldsymbol{V}_{1}$ have to be calculated for a square of $\left(4 N_{b}+1\right) \times\left(4 N_{b}+1\right)$ cylinders, so $N_{c}=$ $\left(4 N_{b}+1\right)^{2} ; l_{x}^{i}, l_{x}^{j}, l_{y}^{i}, l_{y}^{j}=-2 N_{b}, \ldots, 2 N_{b}$ in (45) and (46). By definition, $I_{00}\left(l_{x}, l_{y}\right)=0$ if $\left|l_{x}\right|>N_{b}$ or $\left|l_{y}\right|>N_{b}$. This proves that although only $I$ is known, the updates to obtain all the necessary elements of $\tilde{\boldsymbol{Z}}$ can be computed quickly without ever having to solve a new linear system.

\section{EXAMPLES}

All of the examples presented below involve ECs composed of dielectric cylinders with constitutive parameters $\left(\epsilon_{2}, \mu_{2}\right)=$ $\left(11.56 \epsilon_{0}, \mu_{0}\right)$ and radius $r=0.18 a$ that are arranged on a Cartesian lattice with $\Delta_{x}=\Delta_{y}=a-a$ termed the lattice constant. The cylinders reside in free space, viz., $\left(\epsilon_{1}, \mu_{1}\right)=\left(\epsilon_{0}, \mu_{0}\right)$. This EC has a $\mathrm{TM}_{z}$ bandgap that extends from $k_{1}=0.604(\pi / a)$ to $k_{1}=0.886(\pi / a)$. All calculations are carried out in Matlab on a 2-GHz PC; the multifrontal package used to solve system (23) is UMFPACK Version 4.3 [22].

\section{A. Green Function}

As outlined in Section III-B, the EC Green function is calculated by considering a centrally excited finite EC comprised of $\left(2 N_{b}+1\right) \times\left(2 N_{b}+1\right)$ cylinders with $N_{b}$ large enough to render the Green function vanishingly small beyond the finite EC boundaries. Fig. 9 demonstrates the exponential decay of the EC Green function $\left|\tilde{G}_{0}\left(\boldsymbol{\rho}=x \boldsymbol{u}_{x}\right)\right|$ with $x$ for different frequencies inside the bandgap. The decay rate is larger for 




Fig. 9. EC Green function.

frequencies near the bandgap center than for frequencies near its edge. For example, for $k_{1}=0.74(\pi / a),\left|\tilde{G}_{0}\left(\boldsymbol{\rho}=x \boldsymbol{u}_{x}\right)\right|$ decreases by 11 orders of magnitude from the spatial origin to $x=25 a$. In contrast, for $k_{1}=0.61(\pi / a)$ and $k_{1}=0.88(\pi / a)$, the EC Green function decays only by five orders of magnitude from the spatial origin to $x=25 a$. It suffices to focus on $\left|\tilde{G}_{0}\left(\boldsymbol{\rho}=x \boldsymbol{u}_{x}\right)\right|$ to determine the Green function decay rate as the rate is even faster for $n \neq 0$. All examples reported below involve frequencies near the bandgap center.

For $K=1$ using $N_{b}=15$ with an $N_{s}=3$ shielded-block preconditioner to accelerate convergence of the Matlab build-in BICGSTAB solver, the computation of the EC Green function takes just over $4 \mathrm{~s}$.

\section{B. Eigenmodes}

As described in Section III-C, the EC Green function can be used to characterize the propagating modes of an EC waveguide. Fig. 10 shows the cross-sectional profile of the sole propagating mode in a waveguide that results upon removing one row of cylinders from the EC, obtained by using the proposed scheme with increasing values of $K$; convergence is reached for $K=1$. This waveguide and $K$ value are used for all other examples considered below. Fig. 11 shows the propagation coefficient $\beta$ as a function of angular frequency for the propagating mode of this waveguide.

\section{EC Waveguide Couplers}

The performance and accuracy of the proposed scheme are demonstrated via its application to the analysis of two EC waveguide couplers.

First, consider the ultracompact wavelength multiplexerdemultiplexer depicted in Fig. 3 [4], [5]. The device comprises two identical and coupled EC waveguides that are separated by two rows of cylinders and that jointly support even and odd propagating modes. Fig. 11 shows the dispersion curves of these even and odd modes' propagation constants $\beta_{e}$ and $\beta_{o}$. A field injected into one waveguide will couple entirely

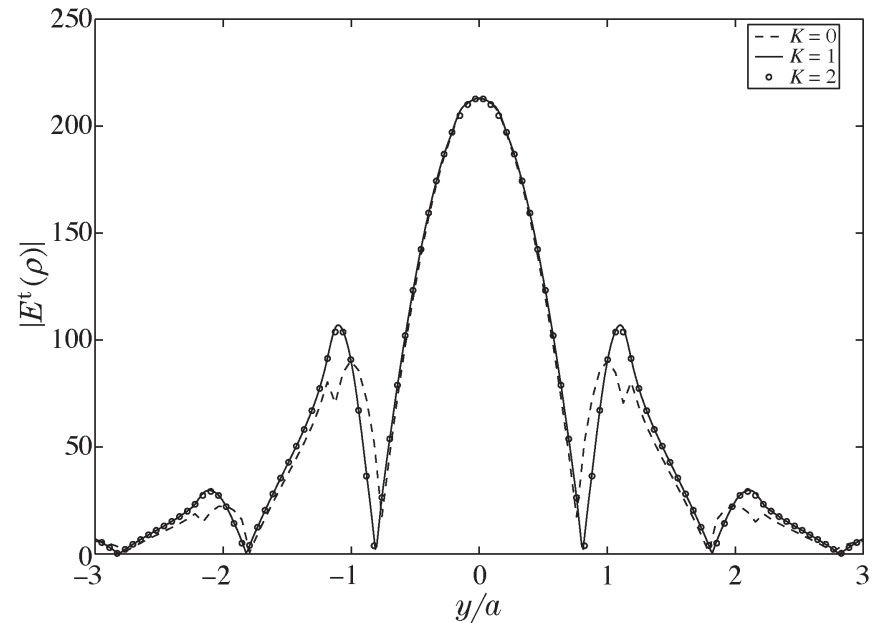

Fig. 10. Mode profile for an increasing value of $K$.

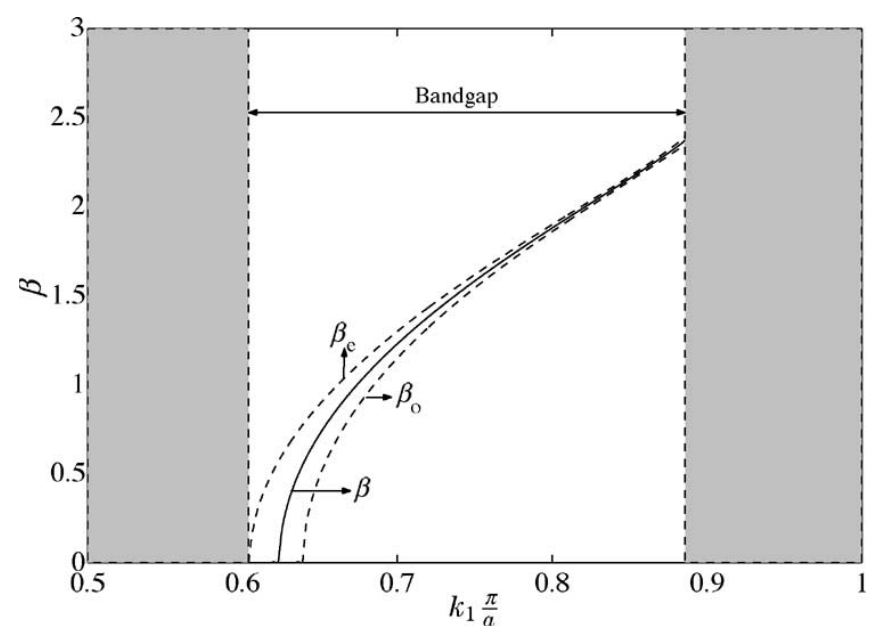

Fig. 11. Dispersion curves: full line, single waveguide; dashed lines, two coupled waveguides.

into the other if the length of the coupling region $l$ is an odd multiple of $L=\left(2 \pi /\left|\beta_{e}-\beta_{o}\right|\right)$. The field however will exit from the output port attached to the input waveguide if $l$ is an integer multiple of $L$. By using the coupled mode theory, the normalized output powers at ports 1, 2, and 3 defined in Fig. 3 are predicted to be

$$
\begin{aligned}
& P_{1}=0 \\
& P_{2}=\sin ^{2}(\kappa l) \\
& P_{3}=\cos ^{2}(\kappa l)
\end{aligned}
$$

with $\kappa=(\pi / L)=\left(\left|\beta_{e}-\beta_{o}\right| / 2\right)$. The transmission spectrum calculated with coupled mode theory for a coupling length $l=1200 a$ is shown in Fig. 12, assuming that $a=540 \mathrm{~nm}$. Unfortunately, the coupled mode theory cannot account for reflections from the $90^{\circ}$ waveguide bends. Fig. 13 shows the same transmission spectra but now calculated using the 


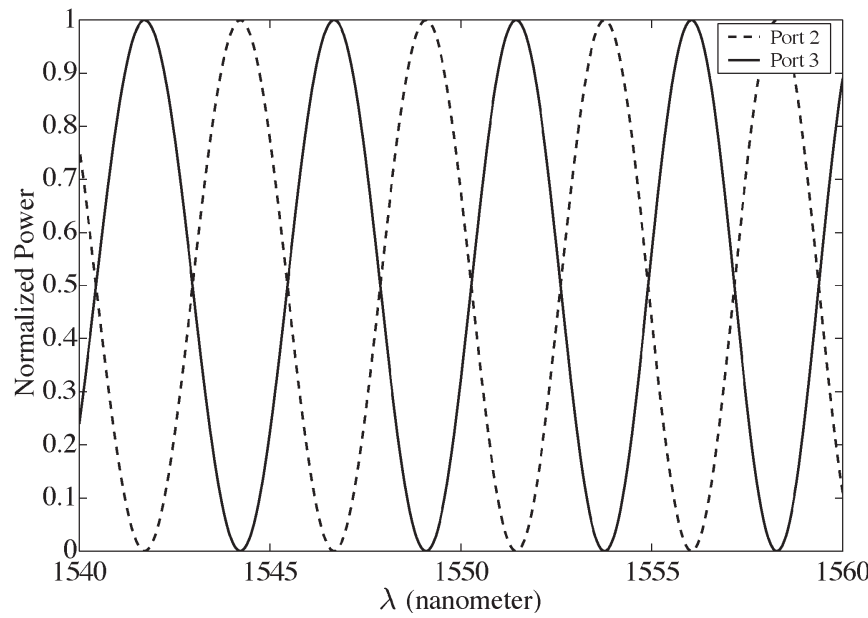

Fig. 12. Transmission spectrum of an EC coupler with $l=1200 a$ calculated with the coupled-mode theory.

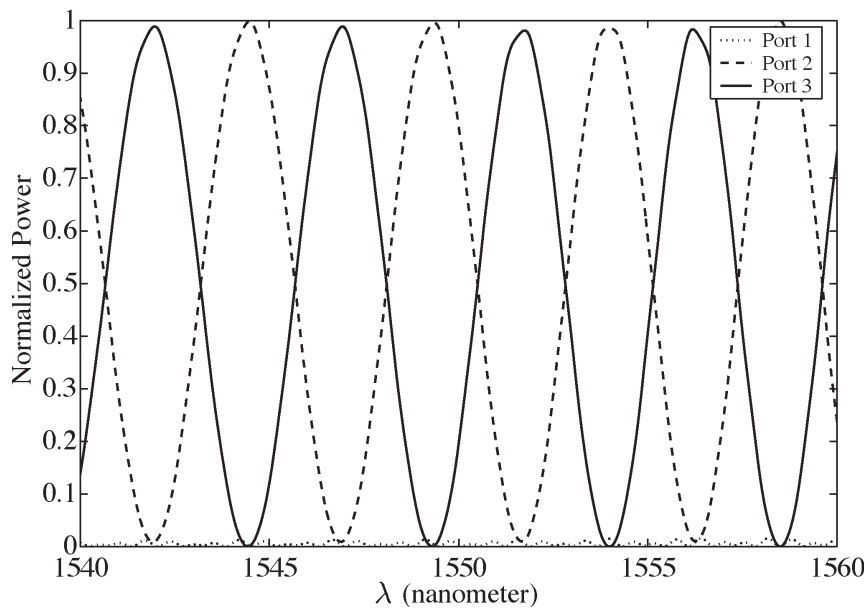

Fig. 13. Transmission spectrum of an EC coupler with $l=1200 a$ calculated with the new scheme.

proposed scheme, which does account for these reflections. Note, for example, that the output power for port 1 is predicted to differ from zero. This example calls for 7465 unknown equivalent electric currents. For one frequency, it takes $12.5 \mathrm{~s}$ to fill the sparse interaction matrix (by selecting pertinent elements out of the precomputed Green function matrix) and $3.8 \mathrm{~s}$ to solve the system of equations (23). The total time to analyze the device, inclusive of the Green function calculation, therefore is just over $20 \mathrm{~s}$. Next, assume that the same device were analyzed using the classical scheme described in Section II. Even if all EC waveguides were lined by only five rows of cylinders to prevent leakage, such analysis would call for 47262 unknowns. Not surprisingly, the CPU times required to solve the system of equations (12) are several orders of magnitude larger than those for the new scheme, even if a fast matrix-vector multiplication method is used.

Second, consider the four-channel multiplexer-demultiplexer depicted in Fig. 14. The various waveguide coupling lengths are $l_{1}=41 a, l_{2}=22 a$, and $l_{3}=24 a$. Using the

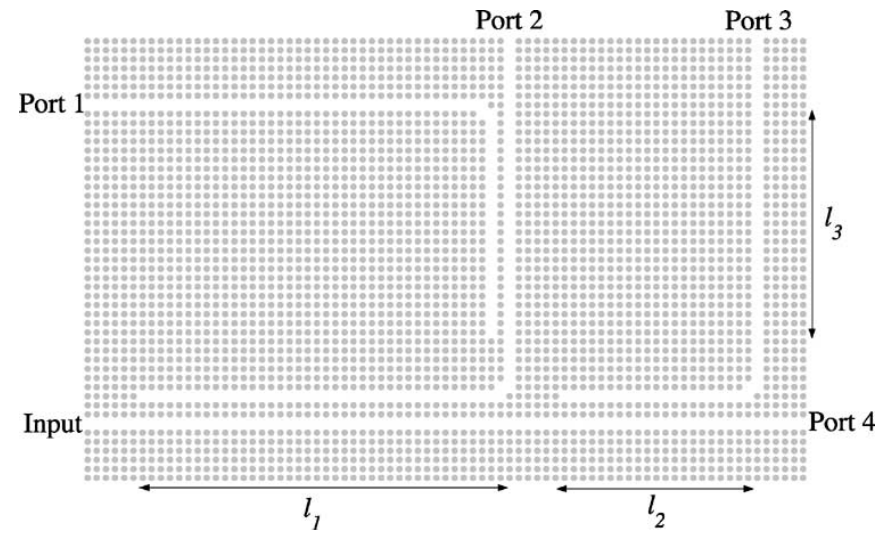

Fig. 14. Four-channel multiplexer-demultiplexer.

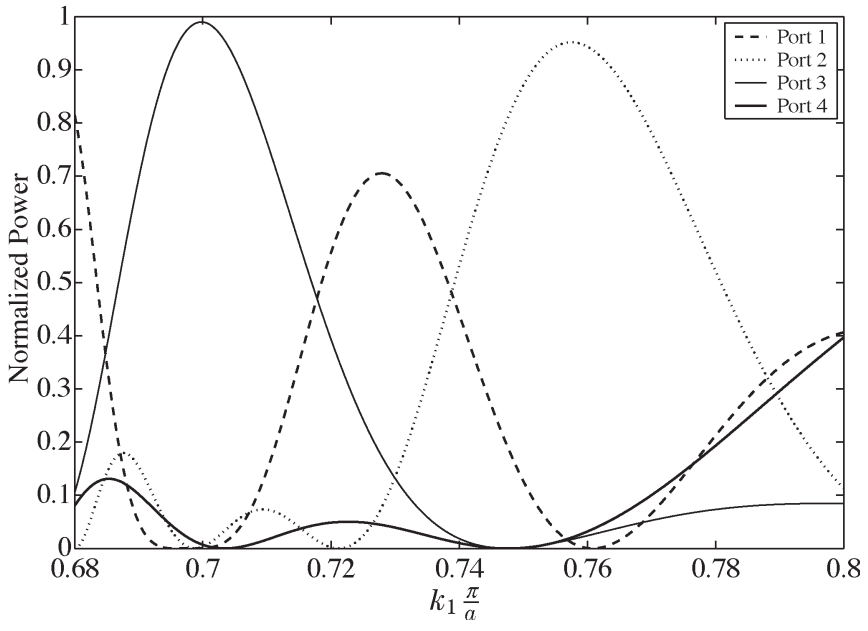

Fig. 15. Transmission spectrum of the four-channel multiplexer-demultiplexer calculated with the coupled mode theory.

coupled mode theory, the normalized output powers at ports 1 , 2, 3, and 4 defined in Fig. 14 are expressed as

$$
\begin{aligned}
& P_{1}=\sin ^{2}\left(\kappa_{2} l_{1}\right) \sin ^{2}\left(\kappa_{1} l_{3}\right) \\
& P_{2}=\sin ^{2}\left(\kappa_{2} l_{1}\right) \cos ^{2}\left(\kappa_{1} l_{3}\right) \\
& P_{3}=\cos ^{2}\left(\kappa_{2} l_{1}\right) \sin ^{2}\left(\kappa_{2} l_{2}\right) \\
& P_{4}=\cos ^{2}\left(\kappa_{2} l_{1}\right) \cos ^{2}\left(\kappa_{2} l_{2}\right)
\end{aligned}
$$

with $\kappa_{i}=\left(\left|\beta_{e i}-\beta_{o i}\right| / 2\right)$ and the subscript $i=1$ or 2 , depending in whether one or two rows of cylinders separate the EC guides. Figs. 15 and 16 show the transmission spectra predicted by the coupled-mode theory and the proposed scheme, respectively. The proposed scheme uses 1066 unknowns to describe fields on removed cylinders. It takes only $2 \mathrm{~s}$ to fill in the interaction matrix (by selecting pertinent elements out of the precomputed Green function matrix) and $0.6 \mathrm{~s}$ to solve the system of equations (23). The total time to analyze the device, inclusive of the Green function calculation, therefore is just over $6.6 \mathrm{~s}$. If the EC waveguides are lined by five rows of cylinders on both sides, this device requires 4638 unknowns when modeled using the classical scheme of 


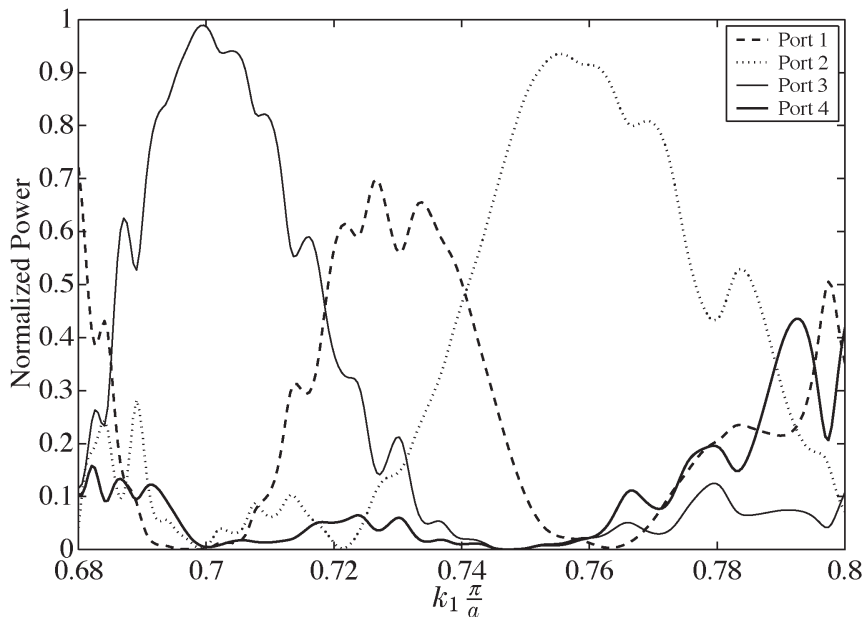

Fig. 16. Transmission spectrum of the four-channel multiplexer-demultiplexer calculated with the new scheme.



Fig. 17. EC filter.

Section II. Using a shielded-block preconditioner, the solution of the system of equations (12) with BICGSTAB to a tolerance of $10^{-4}$ requires 345 iterations and takes $480 \mathrm{~s}$.

\section{Other Defects}

To demonstrate the usefulness of the theory of Section III-D, consider the EC waveguide filter depicted in Fig. 17 [18]. The filter comprises a waveguide that is loaded by two or three cylinders and transmits only signals in a narrow band of frequencies in which the load cylinders jointly resonate. Two configurations were simulated. In the first one, the obstruction consists of two (outer) cylinders separated by a cavity; in the second one, an extra (central) cylinder fills the cavity. The constitutive parameters of the outer and extra cylinders, and the radii of the outer cylinders, equal those of the EC cylinders. The radius of the extra cylinder is four times smaller than those of the EC cylinders $\left(r_{\text {extra }}=0.045 a\right)$. Fig. 18 shows the transmission spectra of both configurations calculated with the conventional free-space Green function and the novel EC Green function MSTs; the conventional analysis uses perfectly matched layers to truncate the EC waveguide and mimic semiinfinite waveguide loads [23]. Excellent agreement between both data sets is observed. The new scheme calls for roughly 300 unknowns to describe the fields in the channels and uses 0.25 and $0.3 \mathrm{~s}$ to fill in the interaction matrix and solve the system of equations (23), respectively.

As a final example, consider the EC waveguide bend depicted in Fig. 19. To minimize reflections, one of the cylinders in the bend is displaced by $\Delta_{x}=0.15 a$ and $\Delta_{y}=-0.15 a$. Fig. 20

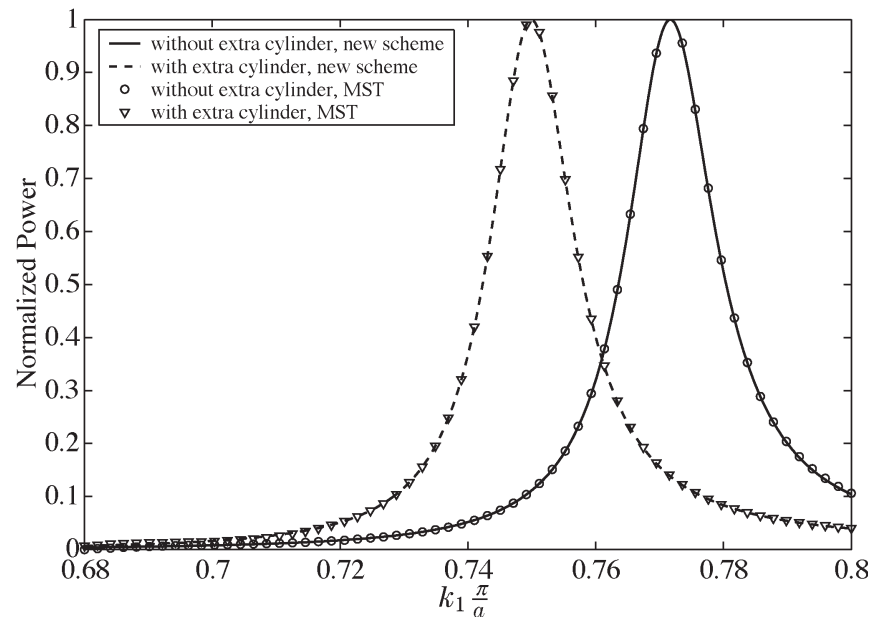

Fig. 18. Transmission spectrum of the EC filter.



Fig. 19. EC bend.

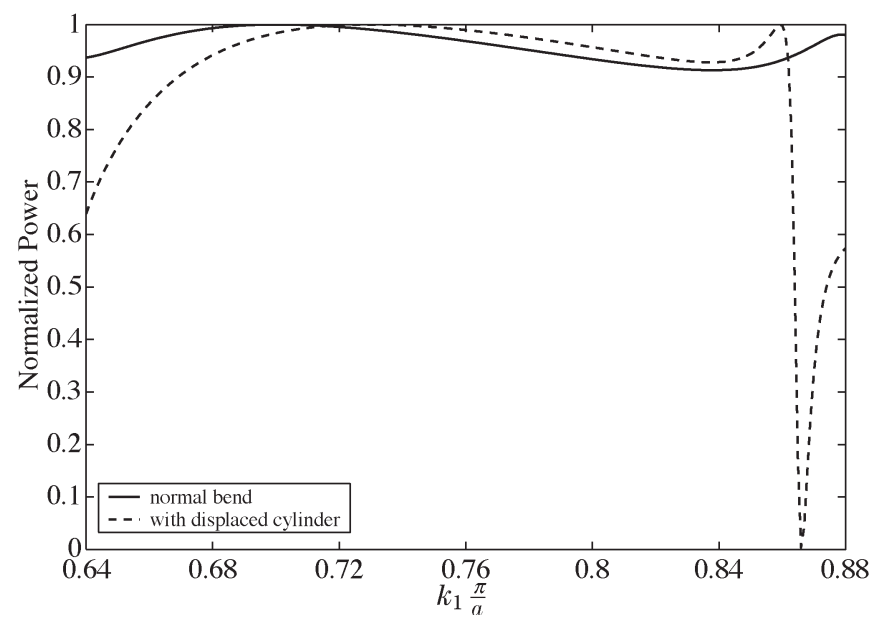

Fig. 20. Transmission spectrum of the EC bend.

shows the transmission spectra of the bend with and without displaced cylinder, respectively. Both configurations require 305 unknown currents, $6.27 \mathrm{~s}$ to fill in the interaction matrix, and $0.5 \mathrm{~s}$ to solve system (23). 


\section{CONCLUSION}

A novel method for simulating wave propagation in twodimensional (2-D) electromagnetic crystal (EC) devices that, a small number of irregular defects aside, are obtained by removing cylinders from infinite doubly periodic and defectless ECs was presented. Integral equations in terms of equivalent currents that reside on the surfaces of removed cylinders were constructed by using Green functions innate to the defectless EC or low-rank perturbations thereof. The solution of the sparse system of equations that resulted upon discretizing these integral equations was effected using a multifrontal method. The scheme was shown to be ideally suited for extracting EC device $S$ parameters, as it permits imposing modal excitations and exact boundary conditions. Although this paper only considered EC devices defined on a square Cartesian lattice that supports $\mathrm{TM}_{z}$-polarized fields, extensions of the proposed scheme to EC devices defined on a hexagonal lattice and/or supporting $\mathrm{TE}_{z}-$ polarized fields are trivial. ECs formed by noncircular scatterers can be treated as well by characterizing their interactions via scattering matrices.

\section{REFERENCES}

[1] J. D. Joannopoulos, R. D. Meade, and J. N. Winn, Photonic Crystals, Molding the Flow of Light. Princeton, NJ: Princeton Univ. Press, 1995.

[2] A. Mekis, J. C. Chen, I. Kurland, S. Fan, P. R. Villeneuve, and J. D. Joannopoulos, "High transmission through sharp bends in photonic crystal waveguides," Phys. Rev. Lett., vol. 77, no. 18, pp. 3787-3790, Oct. 1996.

[3] H. Benisty, C. Weisbuch, M. Agio, M. Kafesaki, C. M. Soukoulis, M. Qiu, M. Swillo, A. Karlsson, B. Jaskorzynska, A. Talneau, J. Moosburger, M. Kamp, A. Forchell, R. Ferrini, R. Houdré, and U. Oesterle, "Models and measurements for the transmission of submicronwidth waveguide bends defined in two-dimensional photonic crystals," IEEE J. Quantum Electron., vol. 38, no. 7, pp. 770-785, Jul. 2002.

[4] M. Koshiba, "Wavelength division multiplexing and demultiplexing with photonic crystal waveguide couplers," J. Lightw. Technol., vol. 19, no. 12, pp. 1970-1975, Dec. 2001.

[5] S. Boscolo, M. Midrio, and C. G. Someda, "Coupling and decoupling of electromagnetic waves in parallel $2 \mathrm{~d}$ photonic crystal waveguides," IEEE J. Quantum Electron., vol. 1, no. 38, pp. 47-53, Jan. 2002.

[6] C. Jin, S. Han, X. Meng, B. Cheng, and D. Zhang, "Demultiplexer using directly resonant tunneling between point defects and waveguides in a photonic crystal," J. Appl. Phys., vol. 91, no. 7, pp. 4771-4773, Apr. 2002.

[7] L. Wu, M. Mazilu, T. Karle, and T. F. Krauss, "Superprism phenomena in planar photonic crystals," IEEE J. Quantum Electron., vol. 38, no. 7, pp. 915-918, Jul. 2002.

[8] A. Taflove, Computational Electrodynamics: The Finite-Difference Time Domain Method. Norwood, MA: Artech House, 1995.

[9] B. Denecker, F. Olyslager, L. Knockaert, and D. De Zutter, "Generation of fdtd subcell equations by means of reduced order modelling," IEEE Trans. Antennas Propag., vol. 51, no. 8, pp. 1806-1817, Aug. 2003.

[10] H. Derudder, D. De Zutter, and F. Olyslager, "A new way to analyse waveguide discontinuities using perfectly matched layers," IEE Electron. Lett., vol. 34, no. 22, pp. 2138-2140, Oct. 1998.

[11] P. Bienstman and R. Baets, "Optical modelling of photonic crystals and VCSELs using eigenmode expansion and perfectly matched layers," Opt. Quantum Electron., vol. 33, no. 4-5, pp. 327-341, Apr. 2001.

[12] G. Tayeb and D. Maystre, "Rigorous theoretical study of finite-size twodimensional photonic crystals doped by microcavities," J. Opt. Soc. Amer. A, vol. 14, no. 12, pp. 3323-3332, Dec. 1997.

[13] D. Pissoort, D. De Zutter, and F. Olyslager, "Efficient semi-analytical analysis of two-dimensional photonic crystals," in IEEE AP-S Int. Symp., Columbus, USA, Jun. 2003, vol. 3, pp. 994-997.

[14] W. C. Chew, J. M. Jin, E. Michielssen, and J. Song, Fast and Efficient Algorithms in Computational Electromagnetics. Boston, MA: Artech House, 2001.
[15] D. Pissoort, D. Vande Ginste, F. Olyslager, and E. Michielssen, "Different preconditioning techniques for the efficient simulation of 2D EC structures," in IEEE AP-S Int. Symp., Monterey, USA, Jun. 2004, vol. 2, pp. 2131-2134.

[16] R. E. Collin, Field Theory of Guided Waves. New York: IEEE Press, 1991.

[17] R. F. Harrington, Time Harmonic Electromagnetic Fields. New York: McGraw-Hill, 1961.

[18] D. Pissoort, B. Denecker, P. Bienstman, F. Olyslager, and D. De Zutter, "Comparative study of three methods for the simulation of twodimensional photonic crystals," J. Opt. Soc. Amer. A, vol. 21, no. 11, pp. 2186-2195, Nov. 2004.

[19] I. S. Duff and J. K. Reid, "The multifrontal solution of unsymmetric sets of linear equations," SIAM J. Sci. Comput., vol. 5, no. 3, pp. 633-641, Sep. 1984.

[20] R. S. Cheng, D. X. Wang, E. K. N. Yung, and J. M. Jin, "Application of the multifrontal method to the vector FEM for analysis of microwave filters," Microw. Opt. Technol. Lett., vol. 31, no. 6, pp. 465-470, Oct. 2001.

[21] G. H. Golub and C. F. Van Loan, Matrix Computations. Baltimore, MD: The Johns Hopkins Univ. Press, 1983.

[22] http://www.cise.ufl.edu/davis/.

[23] D. Pissoort and F. Olyslager, "Termination of periodic waveguides by pmls in time-harmonic integral equation like techniques," Antennas Wireless Propag. Lett., vol. 2, pp. 281-284, 2003.

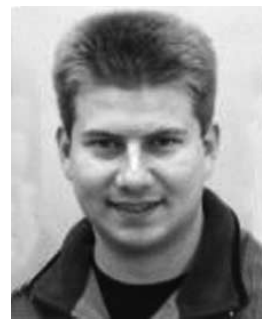

Davy Pissoort was born in 1978 . He received the M.Sc. degree in electrical engineering and the $\mathrm{Ph} . \mathrm{D}$. degree from Ghent University, Belgium, in 2001 and 2005, respectively. He is currently working as a Research Assistant of the Fund for Scientific Research-Flanders, Belgium (FWO-Vlaanderen), Ghent University. His current research interests include the analysis of electromagnetic waveguides and electromagnetic crystal devices.

Eric Michielssen (M'95-SM'99-F'02) received the M.S. degree in electrical engineering (summa cum laude) from the Katholieke Universiteit Leuven (KUL), Belgium, in 1987, and the Ph.D. degree in electrical engineering from the University of Illinois at Urbana-Champaign (UIUC), in 1992.

He served as a Research and Teaching Assistant in the Microwaves and Lasers Laboratory, KUL, from 1987 to 1988, and the Electromagnetic Communication Laboratory, UIUC, from 1988 to 1992 . He joined the faculty of the Department of Electrical and Computer Engineering, UIUC, in 1993, where he serves as Professor and is the Associate Director of the Center for Computational Electromagnetics.

Dr. Michielssen is a Member of URSI Commission B, was awarded the UIUC's 2001 Xerox Award for Faculty Research, appointed 2002 Beckman Fellow in the UIUC Center for Advanced Studies, named 2003 Scholar in the Tel Aviv University Sackler Center for Advanced Studies, and selected as UIUC 2003 University and Sony Scholar. He also served as the Technical Chairman of the 1997 Applied Computational Electromagnetics Society (ACES) Symposium (Review of Progress in Applied Computational Electromagnetics, March 1997, Monterey, CA) and served on the ACES Board of Directors (1998-2001 and 2002-2003) and as ACES Vice-President (1998-2001). From 1997 to 1999 , he was as an Associate Editor for Radio Science. He is currently an Associate Editor for the IEEE TRANSACTIONS ON ANTENNAS AND PROPAGATION. 


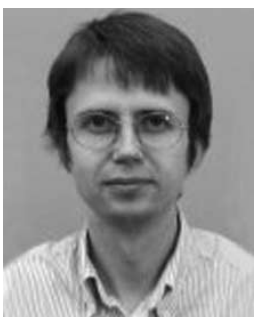

Frank Olyslager (S'90-M'94-SM'99-F'04) was born in 1966. He received both the degree in electrical engineering and the Ph.D. degree from Ghent University, Belgium, in 1989 and 1993, respectively.

$\mathrm{He}$ is the Assistant Secretary General of Union Radio-Scientifique Internationale and was Associate Editor of Radio Science. At present, he is a Full Professor in electromagnetics at Ghent University. His research concerns different aspects of theoretical and numerical electromagnetics. He has authored and coauthored about 160 papers in journals and proceedings.

Dr. Olyslager became laureate of the Royal Academy of Sciences, Literature and Fine Arts of Belgium in 1994. He received the 1995 IEEE Microwave Prize for the best paper published in the 1993 IEEE TRANSACTIONS ON Microwave Theory AND Techniques and the 2000 Best Transactions Paper Award for the best paper published in the 1999 IEEE TRANSACTIONS ON EleCtromagnetic COMPATIBILITY. In 2002, he received the Issac Koga Gold Medal at the URSI General Assembly.

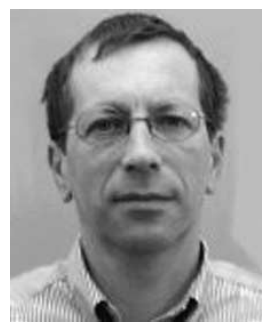

Daniël De Zutter (M'92-SM'96-F'01) was born in 1953. He received the M.Sc. degree in electrical engineering from the University of Ghent, Gent, Belgium, in 1976, the Ph.D. degree and German Habilitation from the University of Ghent in 1981, and completed a thesis leading to a degree equivalent to the French Aggrégation or the German Habilitation in 1984.

From 1976 to 1984 , he was a Research and Teaching Assistant at the University of Gent. From 1984 to 1996 , he was with the National Fund for Scientific Research of Belgium. He is now a Full Professor of electromagnetics. His current research focuses on all aspects of circuit and electromagnetic modeling of high-speed and high-frequency interconnections and packaging on electromagnetic compatibility and numerical solutions of Maxwell's equations. $\mathrm{He}$ has authored and coauthored more than 140 international journal papers and 150 papers in conference proceedings.

Dr. De Zutter was elected a Member of the Electromagnetics Society in 1990. He received the 1990 Montefiore Prize of the University of Liège and the 1995 IEEE Microwave Prize Award (with F. Olyslager and K. Blomme) from the IEEE Microwave Theory and Techniques Society for best publication in the field of microwaves for the year 1993. In 1999, he received the Transactions Prize Paper Award from the IEEE EMC Society. 